\title{
A Spatially Resolved Study of Cold Dust, Molecular Gas, H II Regions, and Stars in the $z=2.12$ Submillimeter Galaxy ALESS67.1
}

\author{
Chian-Chou Chen (陳建州) $)^{1,2}$ (1), J. A. Hodge ${ }^{3}$ (1) Ian Smail ${ }^{2}$ (1) , A. M. Swinbank ${ }^{2}$ (1), Fabian Walter ${ }^{4}$ (1), J. M. Simpson ${ }^{5}$ (1), \\ Gabriela Calistro Rivera $^{3}$, F. Bertoldi ${ }^{6}$ (D), W. N. Brandt ${ }^{7,8,9}$, S. C. Chapman ${ }^{10}$, Elisabete da Cunha ${ }^{11}$, H. Dannerbauer ${ }^{12,13}$ (D), \\ C. De Breuck ${ }^{1}$ (D) C. M. Harrison ${ }^{1}$ (D), R. J. Ivison ${ }^{1,14}$ (D) A. Karim ${ }^{6}$ (D) K. K. Knudsen ${ }^{15}$ (D), \\ J. L. Wardlow ${ }^{2}$ (D) A. Weiß ${ }^{16}$ (D) and P. P. van der Werf ${ }^{3}$ (i) \\ ${ }^{1}$ European Southern Observatory, Karl Schwarzschild Strasse 2, Garching, Germany; ccchen@eso.org \\ ${ }^{2}$ Centre for Extragalactic Astronomy, Department of Physics, Durham University, South Road, Durham DH1 3LE, UK \\ ${ }^{3}$ Leiden Observatory, Leiden University, P.O. Box 9513, 2300 RA Leiden, the Netherlands \\ ${ }^{4}$ MaxPlanck Institut für Astronomie, Königstuhl 17, D-69117 Heidelberg, Germany
${ }_{\text {Academia Sinica Institute of Astronomy and Astrophysics, No. 1, Sec. 4, Roosevelt Road, Taipei 10617, Taiwan }}$ \\ ${ }^{6}$ ArgelanderInstitute of Astronomy, Bonn University, Auf dem Hügel 71, D53121 Bonn, Germany \\ ${ }^{7}$ Department of Astronomy \& Astrophysics, 525 Davey Lab, Pennsylvania State University, University Park, PA 16802, USA \\ ${ }^{8}$ Institute for Gravitation and the Cosmos, Pennsylvania State University, University Park, PA 16802, USA \\ ${ }^{9}$ Department of Physics, 104 Davey Lab, The Pennsylvania State University, University Park, PA 16802, USA \\ ${ }^{10}$ Department of Physics and Atmospheric Science, Dalhousie University, Halifax, NS B3H 3J5, Canada \\ ${ }^{11}$ The Australian National University, Mt Stromlo Observatory, Cotter Road, Weston Creek, ACT 2611, Australia \\ ${ }_{12}$ Instituto de Astrofisica de Canarias (IAC), E-38205 La Laguna, Tenerife, Spain \\ ${ }^{13}$ Universidad de La Laguna, Dpto. Astrofsica, E-38206 La Laguna, Tenerife, Spain \\ ${ }^{14}$ Institute for Astronomy, University of Edinburgh, Royal Observatory, Blackford Hill, Edinburgh EH9 3HJ, UK \\ ${ }^{15}$ Department of Earth and Space Sciences, Chalmers University of Technology, Onsala Space Observatory, SE-439 92 Onsala, Sweden \\ ${ }^{16}$ Max-Planck-Institut für Radioastronomie, Auf dem Hügel 69, D-53121 Bonn, Germany \\ Received 2017 April 14; revised 2017 July 28; accepted 2017 August 3; published 2017 September 7
}

\begin{abstract}
We present detailed studies of a $z=2.12$ submillimeter galaxy, ALESS67.1, using sub-arcsecond resolution ALMA, adaptive optics-aided VLT/SINFONI, and Hubble Space Telescope (HST)/CANDELS data to investigate the kinematics and spatial distributions of dust emission $\left(870 \mu \mathrm{m}\right.$ continuum), ${ }^{12} \mathrm{CO}(J=3-2)$, strong optical emission lines, and visible stars. Dynamical modeling of the optical emission lines suggests that ALESS67.1 is not a pure rotating disk but a merger, consistent with the apparent tidal features revealed in the HST imaging. Our sub-arcsecond resolution data set allows us to measure half-light radii for all the tracers, and we find a factor of 4-6 smaller sizes in dust continuum compared to all the other tracers, including ${ }^{12} \mathrm{CO}$; also, ultraviolet (UV) and $\mathrm{H} \alpha$ emission are significantly offset from the dust continuum. The spatial mismatch between the UV continuum and the cold dust and gas reservoir supports the explanation that geometrical effects are responsible for the offset of the dusty galaxy on the IRX- $\beta$ diagram. Using a dynamical method we derive an $\alpha_{\mathrm{CO}}=1.8 \pm 1.0$, consistent with other submillimeter galaxies (SMGs) that also have resolved $\mathrm{CO}$ and dust measurements. Assuming a single $\alpha_{\mathrm{CO}}$ value we also derive resolved gas and star formation rate surface densities, and find that the core region of the galaxy $(\lesssim 5 \mathrm{kpc})$ follows the trend of mergers on the Schmidt-Kennicutt relationship, whereas the outskirts $\gtrsim 5$ $\mathrm{kpc}$ ) lie on the locus of normal star-forming galaxies, suggesting different star formation efficiencies within one galaxy. Our results caution against using single size or morphology for different tracers of the star formation activity and gas content of galaxies, and therefore argue the need to use spatially resolved, multi-wavelength observations to interpret the properties of SMGs, and perhaps even for $z>1$ galaxies in general.
\end{abstract}

Key words: cosmology: observations - galaxies: evolution - galaxies: formation - galaxies: high-redshift galaxies: star formation - submillimeter: galaxies

\section{Introduction}

Recent technical advances in instruments now allow astronomers to conduct spatially resolved, multi-wavelength observations of astronomical sources. This is particularly important as observations at different wavelengths probe different physical processes, and only by combining the data across many wavelengths is it possible to put together a complete picture of galaxy formation and evolution and draw an unbiased conclusion.

The importance of spatially resolved, multi-wavelength observations is well illustrated in the local universe. Surveys of nearby galaxies in a variety of wavebands have offered great legacy value, including a census of star-forming regions and young stars in the ultraviolet (UV; Gil de Paz et al. 2007) and optical (Gunn et al. 2006), dust distributions in the infrared (IR; Kennicutt et al. 2003, 2011), as well as molecular gas traced in the millimeter by CO (Leroy et al. 2009) and at radio wavelength for atomic hydrogen (Walter et al. 2008). However, it is only by combining these surveys that fundamental insights into galaxy formation, such as the Schmidt-Kennicutt relationship, are revealed (e.g., Kennicutt 1998b; Leroy et al. 2008; Sandstrom et al. 2013).

At high redshifts, however, where observations suffer from cosmological dimming and typically smaller galaxy sizes, obtaining sensitive multi-wavelength data sets on a common galaxy sample becomes difficult. This is particularly true for dust-obscured populations such as submillimeter galaxies (SMGs; Smail et al. 1997; Barger et al. 1998; Hughes et al. 
1998), or more generally the class of dusty star-forming galaxies (DSFGs; Casey et al. 2014a).

SMGs are submillimeter-bright dusty galaxies which are shown to be forming stars at some of the highest rates known, with star formation rates (SFRs) up to $\sim 1000 M_{\odot} \mathrm{yr}^{-1}$ (e.g., Barger et al. 2012; Swinbank et al. 2014). For $850 \mu \mathrm{m}$-selected SMGs they are found to be most prevalent at $z \sim 2-3$ (e.g., Chapman et al. 2005; Wardlow et al. 2011; Simpson et al. 2014; Chen et al. 2016), corresponding to the peak of the cosmic SFR density (Madau \& Dickinson 2014), and they appear to be some of the most massive galaxies existing during that epoch (e.g., Barger et al. 2014). Therefore since their discovery, SMGs have provided an ideal laboratory for testing the physical conditions in which extreme star formation occurs, both theoretically (e.g., Baugh et al. 2005; Davé et al. 2010; Hayward et al. 2013; Cowley et al. 2015) and observationally (e.g., Swinbank et al. 2006; Bouché et al. 2007; Bothwell et al. 2010; Alaghband-Zadeh et al. 2012; Sharon et al. 2013; Rawle et al. 2014; Hodge et al. 2015).

Among the available observational tests, measurements of galaxy dynamics through ionized or molecular gas and the spatial distribution of dust and stars have the most distinguishing power between models (e.g., Narayanan et al. 2010; Bournaud et al. 2014). However, obtaining these data is also the most difficult due to the requirement of high $(\sim 0$ !! 1$)$ spatial resolution. For $z \sim 2$ SMGs in the blank field this can only be achieved with near-IR (NIR) integral field unit (IFU) observations aided by adaptive optics (AO) for redshifted optical emission lines such as $\mathrm{H} \alpha$ (e.g., Alaghband-Zadeh et al. 2012), interferometers to obtain resolved far-IR (FIR)/(sub-)millimeter continuum or CO (e.g., Younger et al. 2008), and space-based observatories such as the Hubble Space Telescope (HST; e.g., Swinbank et al. 2010) to provide diffraction-limited UV-to-NIR imaging of the stellar continuum. The rarity of SMGs means that detailed SMG studies to date have either focused on the UV/optical/NIR (e.g., Menéndez-Delmestre et al. 2013; Chen et al. 2015) or the FIR/ submillimeter (e.g., Danielson et al. 2011; Spilker et al. 2014; ALMA Partnership et al. 2015).

The need to combine UV/optical/NIR and FIR/submillimeter imaging on individual sources is driven by the significant differences sometimes found when comparing results from the two types of study. First, studies of $\mathrm{H} \alpha$ dynamics have found that SMGs are mostly dispersion-dominated systems and are consistent with being mergers (Alaghband-Zadeh et al. 2012; Menéndez-Delmestre et al. 2013; Olivares et al. 2016), whereas the kinematics of $\mathrm{CO}$ and $[\mathrm{C}$ II] on some of the other samples of SMGs have been shown to resemble the structures of rotating disks (e.g., Hodge et al. 2012; De Breuck et al. 2014). While part of this could be the different relaxation timescale between gas and H II regions (e.g., Hopkins et al. 2013), it could also be that the kinematic of $\mathrm{CO}$ and $\mathrm{H} \alpha$ are in fact consistent with each other once measured on the same galaxies, and the different results are genuine variations simply due to small numbers of sources in both types of study.

Such resolved studies would help answer various open questions about SMGs. For example, by compiling a sample of $z<3.5$ DSFGs that have rest-frame UV coverage, Casey et al. (2014b) have found significantly bluer UV continuum slopes $(\beta)$ than the local SFG samples given a fixed IR-to-UV luminosity ratio (IRX). Casey et al. had argued that geometrical effects in which a mismatch between the bulk of IR and UV emissions, which is also observed in local ultra-luminous infrared galaxies (ULIRGs; Sanders \& Mirabel 1996), could be among the most important factors that cause the deviation of DSFGs from the nominal IRX- $\beta$ relationship. By combining high-resolution imaging of the optical and dust emission we can test these hypotheses.

Similarly, by modeling the UV-to-NIR spectral energy distributions (SEDs) it has been found that the dust extinction against the NIR-detectable stellar continuum of SMGs is typically $A_{\mathrm{V}} \sim 1-3$ (e.g., da Cunha et al. 2015), in contrast with the estimates $\left(A_{\mathrm{V}} \sim 500\right)$ based on the column density of dust where the size of the dusty regions is available (Simpson et al. 2017). Although these two studies were conducted using different SMG samples, Simpson et al. argued that the relative compact sizes and distributions of dust with respect to the UV-to-NIR continuum could be the main cause of the discrepancy, simply because the flux-weighted SED modeling based on UV-to-NIR photometry is not reflecting the majority of the dust extinction that is coming from a more compact and very dense and dusty region. These examples illustrate that having spatially resolved panchromatic data with both photometry and spectroscopy on the same galaxies is the key to making further progress on these issues.

Here we present such a study of the $z=2.12$ SMG ALESS67.1, where we have collected a sub-arcsecond UVto-NIR continuum from the HST, NIR IFU from the AO-aided SINFONI observations, and $870 \mu \mathrm{m}$ continuum and ${ }^{12} \mathrm{CO}(J=3-2)$ from the Atacama Large Millimeter/submillimeter Array (ALMA). ALESS67.1 is part of the ALESS sample (Hodge et al. 2013; Karim et al. 2013), a Cycle 0 ALMA survey targeting a flux-limited sample of 126 submillimeter sources detected by a LABOCA (Siringo et al. 2009) $870 \mu \mathrm{m}$ survey in the Extended Chandra Deep Field South (ECDFS) field (LESS survey; Weiß et al. 2009).

ALESS67.1 is one of the few SMGs so far that is covered by all the necessary follow-up observations, and it is representative of the ALESS sample; ALESS67.1 has a spectroscopic redshift at $z_{\mathrm{spec}}=2.1230$ (Danielson et al. 2017) with an SFR of $\sim 500 M_{\odot} \mathrm{yr}^{-1}$ (Swinbank et al. 2014; da Cunha et al. 2015) and a stellar mass of $\sim 2 \times 10^{11} M_{\odot}$ (Simpson et al. 2014; da Cunha et al. 2015). ALESS67.1 appears to be a merger remnant in the HST imaging (Chen et al. 2015), and it is detected by Chandra in the $0.5-2 \mathrm{keV}$ X-ray band (Wang et al. 2013). However, because of its relatively low X-ray luminosity $\left(L_{0.5-8 \mathrm{keV}}=\right.$ $3 \times 10^{42} \mathrm{erg} \mathrm{s}^{-1}$ ), Wang et al. concluded that the X-ray luminosity might be contributed by both star formation and active galactic nuclei (AGNs), which is consistent with the optical line ratios (Danielson et al. 2017), indicating that ALESS67.1 lies in the composite region of the Baldwin, Phillips $\&$ Terlevich (BPT) diagram. Here we include in our analyses the high-resolution ALMA $870 \mu \mathrm{m}$ continuum observations, ${ }^{12} \mathrm{CO}(J=3-2)$, and AO-aided SINFONI. The data reduction and analyses are presented in Section 2 and our results are in Section 3. We discuss in Section 4 the kinematics of $\mathrm{CO}$ and $\mathrm{H} \alpha$, CO-to- $\mathrm{H}_{2}$ conversion factor, the size contrast between dust and other tracers and its implication on the IRX- $\beta$ relationship and the Schmidt-Kennicutt relationship. Finally our conclusions are given in Section 5.

In this paper we assume the Planck cosmology: $H_{0}=$ $67.77 \mathrm{~km} \mathrm{~s}^{-1} \mathrm{Mpc}^{-1}, \Omega_{M}=0.31$, and $\Omega_{\Lambda}=0.69$ (Planck Collaboration et al. 2014). We also assume a Chabrier initial mass function (Chabrier 2003). 


\section{Observations and Data Reduction}

\subsection{ALMA $870 \mu \mathrm{m}$ Continuum}

The ALMA Band 7 data were taken on the 2015 August 11, as part of a Cycle 1 project \#2012.1.00307.S (PI: J. Hodge), which targeted 19 SMGs from the Cycle 0 ALESS survey (Hodge et al. 2013). For a detailed description of the project refer to Hodge et al. (2016).

As in the Cycle 0 ALESS program, the Band 7 data were centered on $344 \mathrm{GHz}(\sim 870 \mu \mathrm{m})$. We used the "singlecontinuum" spectral mode, with $4 \times 128$ dual polarization channels over the $8 \mathrm{GHz}$ bandwidth. The primary beam of the ALMA observations is 17!" 4 at full width at half maximum (FWHM).

The ALMA data were obtained using 46 antennas in an extended configuration (C32-6; maximum baseline of $\sim 1.6 \mathrm{~km}$ ). The bandpass, phase, and flux calibrators were J0522-3627, J0348-2749, and J0334-401, respectively, and the total integration time was approximately eight minutes. The data were taken under good phase stability/weather conditions, with a medium precipitable water vapor (PWV) at zenith of $\sim 0.7 \mathrm{~mm}$.

The $u v$ data were inverse Fourier-transformed using natural weighting to produce the dirty continuum image, which was later deconvolved with a synthesized beam (i.e., the dirty beam) using the CLEAN algorithm. The image was gridded to a pixel scale of $0 . \prime 02$ and a size of 20 " 48 (1024 pixels) per side, covering the primary beam of our observations. The FWHM of the synthesized beam was 0 ". $18 \times 0$ ". 15 $(1.5 \times 1.3 \mathrm{kpc}$ at the redshift of ALESS67.1), with a position angle of $64^{\circ} .8$. The rms noise of the dirty map was $1 \sigma=0.07$ mJy beam ${ }^{-1}$.

\section{2. $A L M A{ }^{12} \mathrm{CO} \mathrm{J}=3-2$}

The ALMA data were taken on the 2015 September 6 as part of project 2013.1.00470.S (PI: J. Hodge), which targets a sample of SMGs to obtain sub-arcsecond resolution CO maps to study the properties of molecular gas (C. Rivera et al. 2017, in preparation). The data were taken in Band 3, with the expected frequency of the redshifted $\mathrm{CO}(J=3-2)$ line $\left(\nu_{\text {rest }}=345.7959899 \mathrm{GHz}\right)$ covered by the upper side band, and using three additional basebands to observe the continuum. We used the lowest-resolution frequency division mode (FDM) mode and averaged over eight channels to maintain adequate resolution while keeping the data rate reasonable. The observations were carried out with 36 antennas in an intermediate configuration (maximum baseline $1.6 \mathrm{~km}$ ) and the maximum recoverable scale was 4". 7 . Standard calibration was used and the total integration time on the target was 29 minutes. Data were reduced using CASA version 4.3.1 and the standard pipeline calibration, with some additional flags applied to address bad antennas or times. Imaging was carried out using CASA version 4.7.0. The $u v$ data were inverse Fourier-transformed using natural weighting to produce both the $3 \mathrm{~mm}$ continuum dirty image and the $\mathrm{CO}$ data cube. There was no detection in the $3 \mathrm{~mm}$ continuum, thus putting a $3 \sigma$ constraint on the $3 \mathrm{~mm}$ continuum flux of $S_{3 \mathrm{~mm}} \leqslant 0.054 \mathrm{mJy}$. The $\mathrm{CO}$ cube was gridded into $48 \mathrm{MHz}$ per channel $\left(\sim 130 \mathrm{~km} \mathrm{~s}^{-1}\right)$ and had an average synthesized beam of 0 " $57 \times 0$ ". 49 . The sensitivity of the CO cube was $0.25 \mathrm{mJy}^{\text {beam }}{ }^{-1}$ per $48 \mathrm{MHz}$ channel.

\subsection{VLT/SINFONI}

AO-assisted, IFU observations of the strong optical lines in ALESS 67.1 were taken with the SINFONI IFU between 2013 January and October. At $z=2.12$, the $[\mathrm{N} \mathrm{II}] / \mathrm{H} \alpha$ lines are redshifted to $\lambda \sim 2.05 \mu \mathrm{m}$ and $[\mathrm{O} \mathrm{III}] / \mathrm{H} \beta$ are redshifted to $\lambda \sim 1.55 \mu \mathrm{m}$ so we used the $H K$-band filter and grism which has a spectral resolution of $R=\lambda / \Delta \lambda \sim 5000$, sufficient to separate $\mathrm{H} \alpha$ and the two [N II] lines. Since the low-surface brightness continuum emission is spatially extended across $\sim 3^{\prime \prime}$ in the $H S T H$-band imaging, we used the $8 \times 8^{\prime \prime}$ field of view (FOV) mode of SINFONI. To achieve high spatial resolution, we employed natural guide star AO correction exploiting a nearby bright $(R=12.9 \mathrm{mag})$ star. Each $1 \mathrm{hr}$ observation block (OB) was split in to $4 \times 600 \mathrm{~s}$ exposures, which were dithered by $4^{\prime \prime}$, thus always keeping the target in the FOV. In total, we observed the target for $7.2 \mathrm{ks}$. Data reduction was performed using the ESOREX pipeline, with additional custom routines applied to improve the flat-fielding, sky subtraction, and mosaicing of the cubes. The flux and astrometry calibration was calibrated from the Hawk-I $K$-band imaging. The AO-corrected point-spread function (PSF) had a mean Stehl ratio of 0.3 , ideally corresponding to an angular resolution of $\sim 0$ "! 2 FWHM.

\subsection{HST Optical/NIR Imaging}

The optical and NIR images from the ACS and WFC3 cameras mounted on the HST were taken as part of the Cosmic Assembly Near-IR Deep Extragalactic Legacy Survey (CANDELS; Grogin et al. 2011; Koekemoer et al. 2011). The typical FWHM of the $H S T$ PSF in the optical is $\sim 0$ !' 1 .

\section{Analysis and Results}

\subsection{ALMA $870 \mu$ m Continuиm}

A prominent source is detected in the central region of the high-resolution dirty map, with a peak flux of $0.72 \mathrm{mJy}^{\text {beam }}{ }^{-1}$ (corresponding to $10 \sigma$ ) and a location matching ALESS67.1 from Hodge et al. (2013). We clean a circular region with $1^{\prime \prime}$ radius around the source down to $2 \sigma$, and the resulting cleaned image is shown in Figure 1. As can be seen, two detections were reported at $\sim 1^{\prime \prime}$ resolution observations by Hodge et al. (2013), ALESS67.1/67.2; however, in our data we only detect ALESS67.1. We tried tapering the map to lower spatial resolution to test the possibility that the lack of detection is due to extended structures which are resolved out in the highresolution map. While ALESS67.2 remained undetected in the tapered maps, the sensitivity of the tapered map was not as deep as the original Cycle 0 data so the nature of ALESS67.2 remains inconclusive. It is possible that ALESS67.2 is resolved out, or that it is a false detection.

As ALESS67.1 is clearly resolved and is the sole source detected in the map, to measure the flux and the light profile we first use the UVMODELFIT algorithm to model the $u v$ data. We find that the best-fit (reduced $\chi^{2}=0.5$ ) Gaussian profile has an intensity of $3.7 \pm 0.2 \mathrm{mJy}$ with a FWHM of 0 ". $40 \pm$ 0 " $02 \times 0$ "' $21 \pm 0$ " 02 , corresponding to a physical half-light radius of $1.7 \pm 0.1 \times 0.9 \pm 0.1 \mathrm{kpc}$. We obtain consistent results if we instead use the IMFIT algorithm or SEXTRACTOR on the cleaned image. The measured flux is also consistent with but marginally lower than the previous measurement $(4.9 \pm 0.7 \mathrm{mJy})$ based on the lower-resolution ALMA Cycle 0 

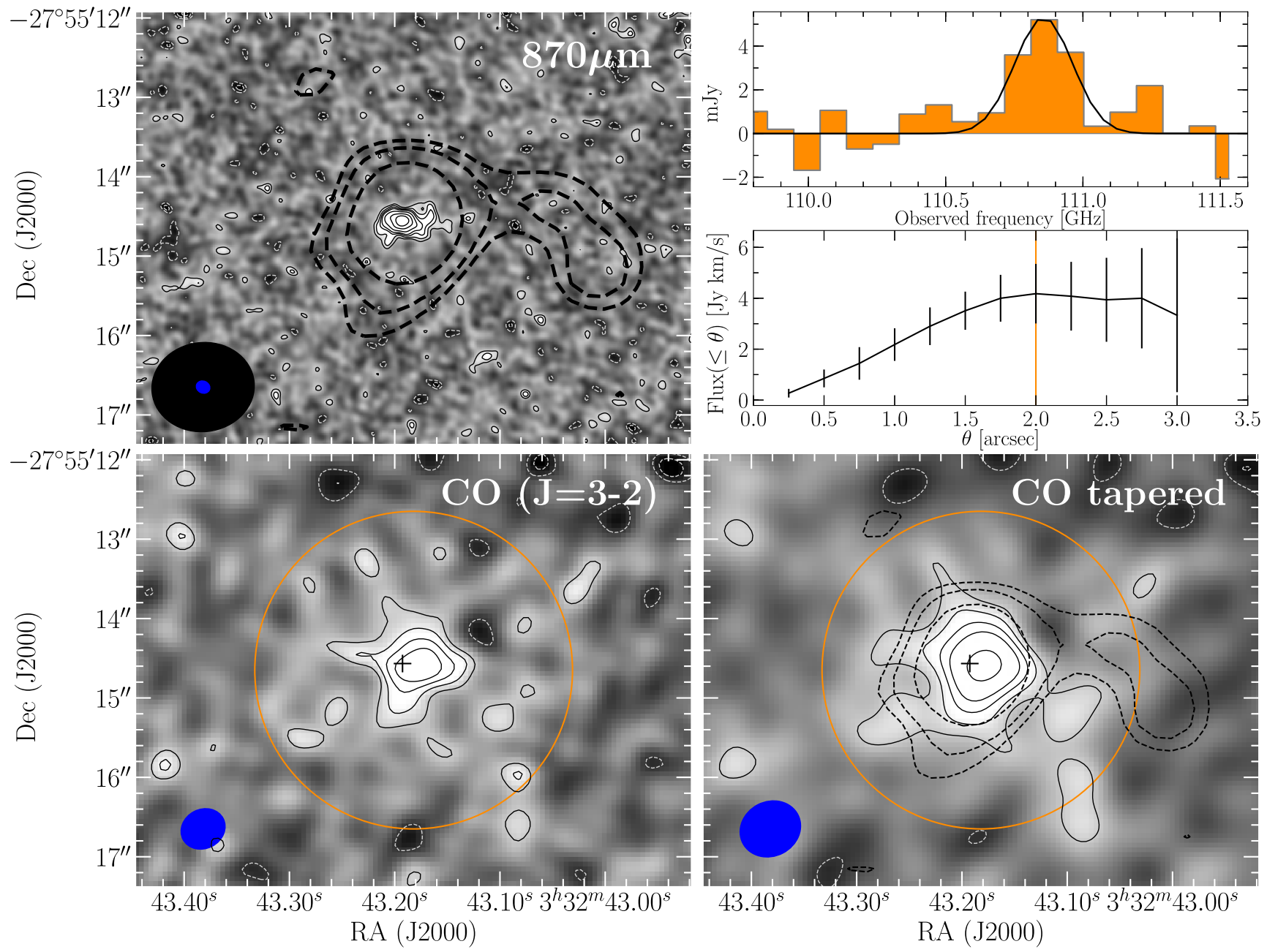

Figure 1. Top left: The ALMA 0". $2870 \mu$ m continuum map with solid contours at levels of $[2,3,4,7,10,13] \times \sigma$. The dotted contours show the detected emission in the $\sim 1^{\prime \prime}$ Cycle 0 ALMA data presented in Hodge et al. (2013), with the levels at $[2,3,5] \times \sigma$. The synthesized beam shapes are shown at the bottom-left corner. Bottom: FWHM-averaged maps of CO based on the spectrum shown in the top-right panel, which is obtained by summing all the fluxes in the naturally weighted map (left) within a $2^{\prime \prime}$ radius circle (orange circles). The radius of $2^{\prime \prime}$ is determined through our curve-of-growth analysis, which is shown below the spectrum. The resolution of the tapered map (right) is $\sim 0$. $^{\prime \prime}$. The solid contours in both maps are $[2,3,4,5] \times \sigma$ and the gray dashed ones are $[-3,-2] \times \sigma$. The dashed contours in the bottom-right panel show the $870 \mu \mathrm{m}$ continuum from the Cycle 0 ALMA data. The small cross symbols mark the peak location of the $870 \mu \mathrm{m}$ continuum emission.

data (Hodge et al. 2013), and the size is consistent with the parent sample of ALESS SMGs with 0!'2 high-resolution ALMA observations (Hodge et al. 2016).

$$
\text { 3.2. } A L M A{ }^{12} \mathrm{CO} \mathrm{J}=3-2
$$

A strong line detection is seen in the dirty $3 \mathrm{~mm}$ channel maps, and the emission appears resolved. To clean the data cube and extract the spectra, we employ the following iterative procedure. We first derive a weight-averaged map over a bestguess frequency width and choose a center based on the averaged map. We then perform a curve-of-growth analysis where we define a circular aperture centered at the chosen centroid with a radius which encompasses all the line flux. The data cube is then cleaned to $2 \sigma$ within this defined aperture and the spectrum is extracted. Next, the extracted spectrum is fitted with a Gaussian profile and the frequency width used for obtaining the averaged map is updated to be the FWHM of the spectral fit. This process continues until the solution converges. Finally we find that all the line flux is contained within a circular radius of $2^{\prime \prime}$ and the results are not sensitive to the chosen position of the aperture center within a beam area.
The results are plotted in Figure 1, showing a line detection well fitted $\left(\chi^{2}=1.1\right)$ with a Gaussian profile centered at $110845 \pm 30 \mathrm{MHz}$ and having a FWHM of $319 \pm 72 \mathrm{MHz}$, corresponding to a ${ }^{12} \mathrm{CO}(J=3-2)$ line at a redshift of $z=2.1196 \pm 0.0009$, with a velocity FWHM of $862 \pm$ $195 \mathrm{~km} \mathrm{~s}^{-1}$. By integrating the best-fit Gaussian we derive a total line flux of $4.2 \pm 1.2 \mathrm{Jy} \mathrm{km} \mathrm{s}^{-1}$. The errors are estimates from the fit, and they are consistent with the errors derived from a Monte Carlo simulation. We create fake spectra by injecting the model profile into spectra extracted from randomly selected regions of the data cube with the same circular aperture used for the detection spectrum. The errors are then obtained from the standard deviations between the fit results and the input model.

Using the standard relation from Solomon \& Vanden Bout (2005), $\quad L_{\mathrm{CO}}^{\prime}=3.25 \times 10^{7} S_{\mathrm{CO}} \Delta v \nu_{\mathrm{obs}}^{-2} D_{\mathrm{L}}^{2}(1+z)^{-3}, \quad$ where $S_{\mathrm{CO}} \Delta v$ is the total line flux in Jy km s${ }^{-1}, \nu_{\mathrm{obs}}$ is the observed line frequency in $\mathrm{GHz}$, and $D_{\mathrm{L}}$ is the cosmological luminosity distance in $\mathrm{Mpc}$, we calculate a $\mathrm{CO}$ luminosity of $L_{\mathrm{CO}(3-2)}^{\prime}=(1.1 \pm 0.3) \times 10^{11} \mathrm{~K} \mathrm{~km} \mathrm{~s}^{-1} \mathrm{pc}^{2}$. Huynh et al. (2017) have recently conducted ${ }^{12} \mathrm{CO}(1-0)$ observations on 
ALESS67.1 using the Australia Telescope Compact Array (ATCA), and they detect strong ${ }^{12} \mathrm{CO}(1-0)$ emission with a total flux of $L_{\mathrm{CO}(1-0)}^{\prime}=(9.9 \pm 1.8) \times 10^{10} \mathrm{~K} \mathrm{~km} \mathrm{~s}^{-1} \mathrm{pc}^{2}$. With both the measurements we calculate a $L_{\mathrm{CO}(3-2)}^{\prime} /$ $L_{\mathrm{CO}(1-0)}^{\prime}$ line luminosity ratio of $r_{3,1}=1.1 \pm 0.4$, consistent with previous estimates for the SMG population (Harris et al. 2010; Ivison et al. 2011; Bothwell et al. 2013; Sharon et al. 2016).

The curve-of-growth analysis shown in Figure 1 suggests a $\mathrm{CO}$ half-light radius of $\sim 1^{\prime \prime}$. To measure the size we employ both image-based and $u v$-based analyses. We first conduct IMFIT on the averaged map and find that the best-fit twodimensional (2D) Gaussian profile has a circularized half-light radius of 0 " $91 \pm 0$ " 16 , consistent with the curve-of-growth analysis. As the best-fit 2D model only has a peak signal-tonoise ratio $(\mathrm{S} / \mathrm{N})$ of 3 , we run the following modeling to estimate the bias and the scatter of our measurement. Random elliptical Gaussian 2D models are first convolved with the synthesized beam and then injected at random positions on the residual averaged $\mathrm{CO}$ map with the best-fit model of the detected signals subtracted. IMFIT is performed on each injected model and the output results are recorded. In total we inject 36,000 models with the peak $\mathrm{S} / \mathrm{N}$, major axis, minor axis, and positional angle all randomized, in which the peak $\mathrm{S} / \mathrm{N}$ has a range of 1-10 and the half-light radius in the major and minor axes is allowed within $2^{\prime \prime}$. We collect input parameters that correspond to an output matching to the $\mathrm{CO}$ measurements in peak $\mathrm{S} / \mathrm{N}$ and circularized radius, and we compare the input and output circularized radius by computing the fractional difference defined as (output-input)/input. We find a 3\% upward bias in median $(0.028 \pm 0.007)$ and a $20 \%$ scatter. The scatter is consistent with the measurement; however, the size bias needs to be corrected. We therefore obtain a bias-corrected ${ }^{12} \mathrm{CO}(J=3-2)$ half-light radius of 0 ". $88 \pm 0$ ". 16 . The deconvolved circularized half-light radius is therefore $0 . " 84 \pm 0$ !" 16 .

For the $u v$-based analysis we extract the averaged visibility over the FWHM channels as a function of the $u v$ distances and then perform $\chi^{2}$ fitting assuming a Gaussian profile. We obtain a half-light radius of 0 " $76 \pm 0$." 10 , in good agreement with the result based on the image-plane analysis. However the $u v$-based measurement is better constrained, with lower errors. We therefore adopt the $u v$-based measurement for the ${ }^{12} \mathrm{CO}$ size. At the measured $\mathrm{CO}$ redshift, the ${ }^{12} \mathrm{CO}$ half-light radius would therefore be $r_{1 / 2, \mathrm{CO}}=6.5 \pm 0.9 \mathrm{kpc}$. More details on the $u v$-fitting will be presented in C. Rivera et al. (2017, in preparation).

\subsection{SINFONI Spectra}

The $\mathrm{H} \alpha$ line is strongly detected in our SINFONI data with an $\mathrm{S} / \mathrm{N} \sim 10$ at the peak, allowing us to derive $2 \mathrm{D}$ intensity, velocity, and dispersion maps. In the following we therefore analyze the spectra in both integrated and 2D. Weaker [N II] and [S II] lines are also detected, although only in the central regions of the source. We also search for [O I]6300, and [O III]/ $\mathrm{H} \beta$ at $\sim 5000 \AA$, but no significant detections are found.

In both $1 \mathrm{D}$ and $2 \mathrm{D}$ cases, we perform a minimizing- $\chi^{2}$ fit to the spectra over a wavelength range of $1.9-2.2 \mu \mathrm{m}$, where the continuum is well described with a power-law slope and covers all the detected lines. The spectra are fit with four Gaussian models, in which all include a linear continuum component with the slope and normalization allowed to be free. We then fit different combination of lines; $\mathrm{H} \alpha, \mathrm{H} \alpha+[\mathrm{N} \mathrm{II}], \mathrm{H} \alpha+[\mathrm{S}$ II $]$, and
$\mathrm{H} \alpha+[\mathrm{N} \mathrm{II}]+[\mathrm{S} \mathrm{II}]$. In all cases we assume that the [N II] and [S II] lines have the same line width and redshift as those of $\mathrm{H} \alpha$, and the flux ratio of the [N II]6583/[N II] 6548 doublet $^{17}$ is fixed to a theoretical value of 3 based on the transition probabilities provided in Osterbrock (1989). The flux ratio of the $[\mathrm{S} \mathrm{II}] 6731 /[\mathrm{S} \mathrm{II}] 6716$ doublet is sensitive to the magnetic field strength, hence it is not fixed. The fits are weighted against the sky spectrum provided by Rousselot et al. (2000) and when calculating $\chi^{2}$ the wavelength ranges corresponding to the skylines are masked. The velocity dispersion is corrected in quadrature for instrumental broadening. The errors are derived using Monte Carlo simulations similar to those used for measuring the errors of the $\mathrm{CO}$ emission. Note that by adding an extra broad Gaussian component we have also searched for broad lines with a FWHM over $1000 \mathrm{~km} \mathrm{~s}^{-1}$, typical for SMGs hosting AGNs and suggesting strong outflows (e.g., Harrison et al. 2012); however, we do not find evidence of such a broad component in ALESS67.1.

The model selection is determined based on the Akaike information criterion (AIC). Specifically, we use the version that is corrected for a finite sample size (AICc; Hurvich \& Tsai 1989), which is defined as AICc $=\chi^{2}+2 k+$ $2 k(k+1) /(n-k-1)$, where $\chi^{2}$ is from the fit, and $k$ and $n$ denote the number of parameters and the number of data, respectively. Normally fits with more model parameters have lower $\chi^{2}$, and therefore a situation of over-fitting may not occur if one simply selects the model that produces the lowest $\chi^{2}$. The AICc offers a quantitative way to compare related models on the goodness of fit by penalizing the number of parameters in the model, and the model that has the lowest AICc is selected as the adopted model in most cases. However, as shown below, [N II]6583 happens to sit on one of the bright skylines, and we find that during the curve-of-growth analyses on the integrated spectra the skyline contamination becomes significant at larger radii. Consequently we restrict the fit to $\mathrm{H} \alpha+[\mathrm{N} \mathrm{II}]+[\mathrm{S}$ II $]$ only.

Finally, a fit with line components is considered significant if the fit, compared to a simple continuum-only model, has a lower AICc and provides a $\chi^{2}$ improvement of $\Delta \chi^{2}>25$, equivalent to an $\mathrm{S} / \mathrm{N}>5 \sigma$ assuming Gaussian noise and that the noise is not correlated among wavelength channels. For models that include [S II], given it is a separated line without skyline contamination, we require a further $\chi^{2}$ improvement of $\Delta \chi^{2}>9(3 \sigma)$ compared to models without [S II].

\subsubsection{Integrated Spectrum}

To determine the radius over which the total flux is measured, we again employ a curve-of-growth approach that is similar to that used for ${ }^{12} \mathrm{CO}$ (see Section 3.2). We adopt the peak position of the $870 \mu \mathrm{m}$ continuum for the centroid, which produces a converged result and lies close to the geometrical center of the 2D intensity distributions shown in the next section. We again move the centroid around within the resolution area and fold the variations into the uncertainties of the measurements. The flux is derived based on the fitting procedure outlined in Section 3.3, except at radii larger than

\footnotetext{
17 The values are air wavelengths and we use these for ease of comparison with the literature. Since SINFONI is situated in a cryo-vacuum chamber in the fitting process we adopt vacuum wavelengths for [O I] at $6302.1 \AA, \mathrm{H} \alpha$ at $6564.7 \AA$, N II doublets at $6550.0 \AA$ and $6585.4 \AA$, and [S II] doublets at $6718.4 \AA$ and $6732.8 \AA$. These values are derived based on the conversion from air to vacuum wavelengths described in Equation (65) of Greisen et al. (2006).
} 
1 ." 5 , where we find that the skyline contamination significantly affects the fit and, by examining the 2D maps, we conclude that the [N II] lines are boosted (since there is no strong emission detected in the 2D map beyond this radius). We therefore fix the peak of the [N II] lines to be that measured at 1 "' 5 but still allow dispersion and redshift to float.

Based on the curve-of-growth approach, the integrated spectra are measured using a circular aperture with a radius of 1 !" 8 , at which all three line fluxes are converged. From this, we measure a redshift of $z=2.1228 \pm 0.0006$, slightly higher than but still consistent with the $\mathrm{CO}$ redshift within $3 \sigma$. We also measure a total $\mathrm{H} \alpha$ flux of $(2.6 \pm 0.4) \times 10^{-16} \mathrm{erg} \mathrm{s}^{-1} \mathrm{~cm}^{-2}$, a [N II] flux of $(1.1 \pm 0.4) \times 10^{-16} \mathrm{erg} \mathrm{s}^{-1} \mathrm{~cm}^{-2}$, and a [S II] flux of $(6.7 \pm 2.9) \times 10^{-17} \mathrm{erg} \mathrm{s}^{-1} \mathrm{~cm}^{-2}$, with a spectral FWHM of $670 \pm 100 \mathrm{~km} \mathrm{~s}^{-1}$. At the measured $\mathrm{H} \alpha+[\mathrm{N} \mathrm{II]}+[\mathrm{S}$ II] redshift we compute a $\mathrm{H} \alpha$ luminosity of $L_{\mathrm{H} \alpha}=(9.2 \pm 1.5) \times 10^{42} \mathrm{erg} \mathrm{s}^{-1}$, a [N II] luminosity of $L_{[\mathrm{N} \mathrm{II]}}=(3.8 \pm 1.4) \times 10^{42} \mathrm{erg} \mathrm{s}^{-1}$, and a [S II] luminosity of $L_{[\mathrm{S} \mathrm{II]}}=(2.4 \pm 1.1) \times 10^{42} \mathrm{erg} \mathrm{s}^{-1}$.

The continuum-subtracted integrated spectra along with the best-fit Gaussian model are shown in Figure 2, in which we also show the best-fit ${ }^{12} \mathrm{CO}(J=3-2)$ profile for comparison. Because the spatial extent of [N II] is much smaller than that of $\mathrm{H} \alpha$, the integrated spectrum with an aperture radius of 1 !" 8 includes extra unnecessary noise and [N II] may appear undetected. To demonstrate that [N II] lines are indeed detected in Figure 2 we also plot the $\mathrm{H} \alpha /[\mathrm{N}$ II] portion of the spectrum with a smaller aperture radius. The line profiles are consistent among the molecular and atomic emission lines, suggesting that in the integrated sense the dynamics that are measured by these tracers agree with each other. We compare in more detail the spatially resolved dynamics between the two tracers in the discussion section.

The curve-of-growth analysis suggests a half-light radius of 0 " $8 \pm 0$ " 1 for $\mathrm{H} \alpha$, which is slightly larger than but consistent with $0 . " 63 \pm 0 . \prime 10$ derived from a best-fit 2D Gaussian profile on the intensity. We adopt the result from the curve-of-growth analysis since the projected $\mathrm{H} \alpha$ emitting area is non-Gaussian with clear extended structures (Figure 3) so a single Gaussian model is likely to underestimate the true size. Given the angular resolution of 0. " 2 of the SINFONI observations, the deconvolved size of $\mathrm{H} \alpha$ is 0 "' $77 \pm 0$ "' 10 , corresponding to a $\mathrm{H} \alpha$ half-light radius of $r_{1 / 2, \mathrm{H} \alpha}=6.6 \pm 0.9 \mathrm{kpc}$ at the $\mathrm{H} \alpha$ redshift. We perform the same curve-of-growth exercise for [N II] and [S II], finding $r_{1 / 2, \mathrm{~N} \mathrm{II}}=5.1 \pm 1.7 \mathrm{kpc}$ and $r_{1 / 2, \mathrm{~S}_{\text {II }}}=5.1 \pm 2.1 \mathrm{kpc}$.

\subsubsection{Two-dimensional Kinematics}

To produce $2 \mathrm{D}$ intensity, velocity, and dispersion maps we run our line-fitting procedures described in Section 3.3 on each spaxel. However, not every spaxel has significant line emission so we adopt an adaptive binning approach that is typically used for high-redshift IFU data (e.g., Swinbank et al. 2006). We start with one spaxel, and if the fit is not significant then we average over $3 \times 3$ spaxels, and if that is still not significant then we increase the binning to $5 \times 5$ spaxels. In regions where this adaptive binning process still fails after $5 \times 5$ binning to give an adequate $\mathrm{S} / \mathrm{N}$, we leave the spaxel without a fit. The caveat of this approach is that the signals are weighted toward the higher $\mathrm{S} / \mathrm{N}$ pixels.

The results are plotted in Figure 3, showing the 2D intensity maps for $\mathrm{H} \alpha,[\mathrm{NII}]$, and [S II]. The velocity and velocity dispersion maps are also shown.

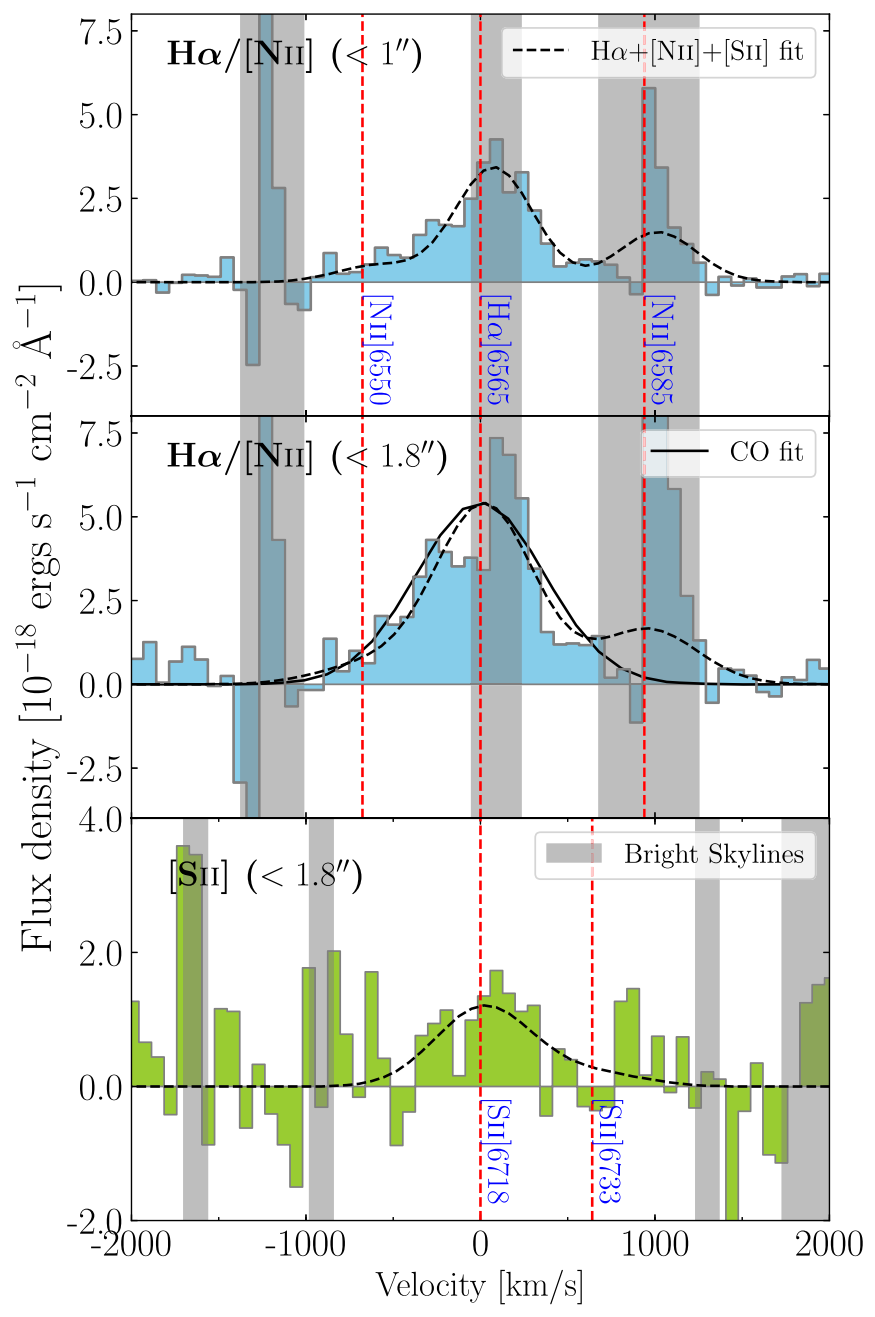

Figure 2. Integrated line profiles for $\mathrm{CO}, \mathrm{H} \alpha /[\mathrm{N} \mathrm{II}]$, and [S II]. The top panel shows the integrated spectrum with an aperture radius of $1^{\prime \prime}$, in order to clearly show the detection of [N II], and the remaining two panels show the spectra with an aperture radius of $1 . " 8$, adopted based on the curve-of-growth analyses in which all the line fluxes are converged. The systemic velocity is referenced at $\mathrm{CO}$ and optical line redshifts. The gray vertical bands mark the positions of the bright sky lines, and the relative line positions are also marked according to their wavelengths. The best-fit CO Gaussian profile is shown as the solid black curve in the middle panel, and the best-fit line profiles for the optical lines are shown as dashed curves. The line profiles are consistent among the molecular and atomic emission lines, suggesting that in the integrated sense the dynamics that are measured by these tracers agree with each other.

The first and most striking feature is how most of the $\mathrm{H} \alpha$ and dust emission are not co-located, with $\mathrm{H} \alpha$ much more extended than the dust by a factor of $\sim 3$. The sky separation between the peaks of the $\mathrm{H} \alpha$ and $870 \mu \mathrm{m}$ continuum is 0 ." 4 , more than $3 \sigma$ given $\sim 0$ !" 2 resolution in FWHM for both the ALMA and SINFONI observations. Although the systematic uncertainty in SINFONI astrometry could contribute to a further offset of $\sim 0$.' 2-0." 3, later we show that the cold dust emission coincides with the regions with the reddest colors revealed by the WFC3 imaging, and most of the $\mathrm{H} \alpha$ emission matches the location of the brightest continuum in the rest-frame optical WFC3 maps. Therefore we conclude that the apparently disjoint nature between the cold dust as traced by the $870 \mu \mathrm{m}$ continuum and the $\mathrm{H} \alpha$ emission is genuine. However, on the other hand, we find that the sky locations of $[\mathrm{N} \mathrm{II}]$ and $[\mathrm{S} \mathrm{II}]$ peak at the position of the dust emission, although both [N II] and [S II] are slightly more extended than the dust. The enhanced 

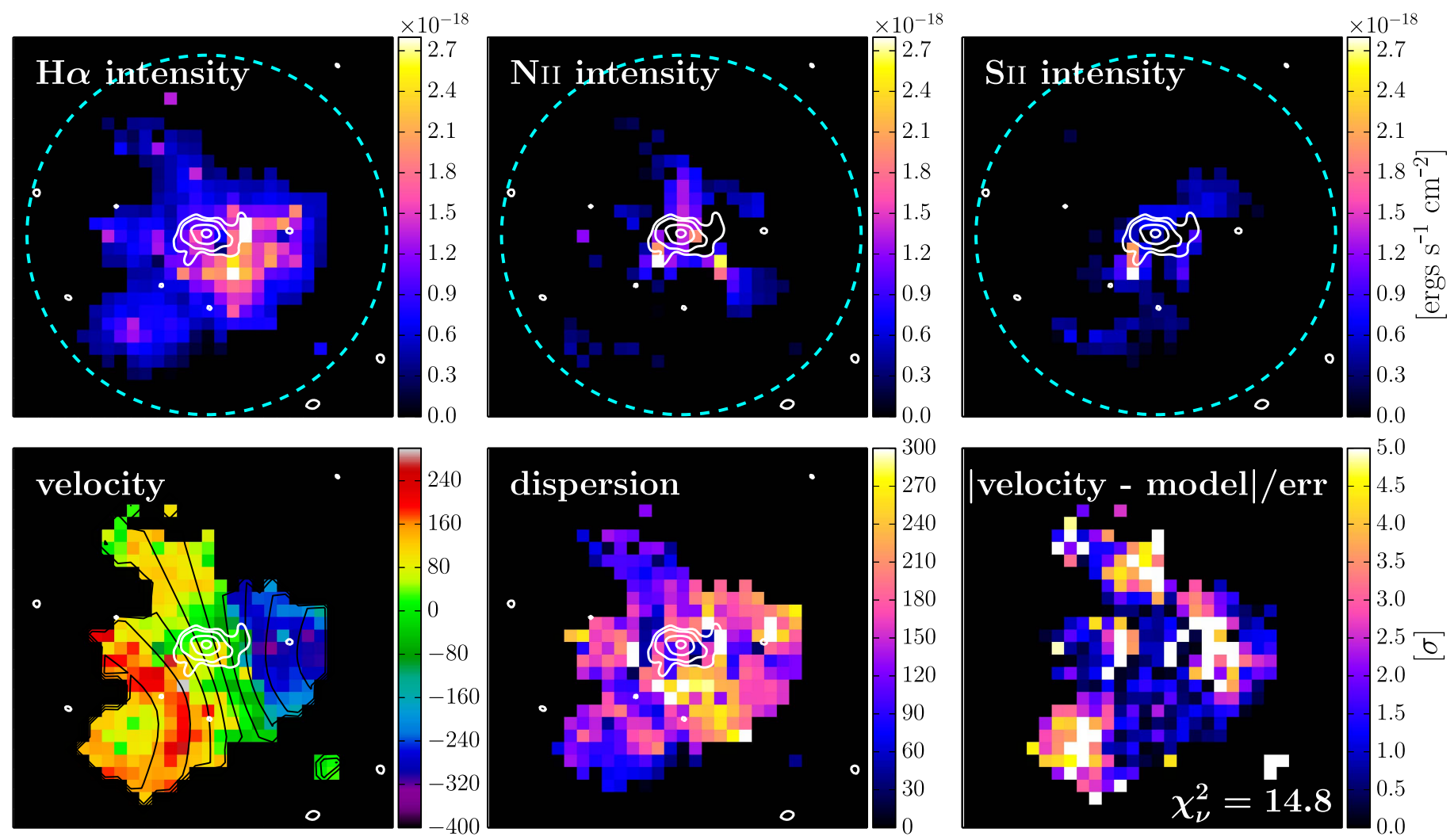

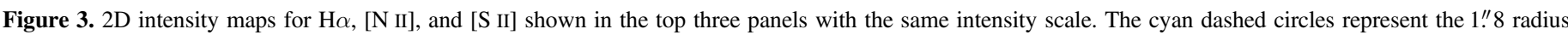

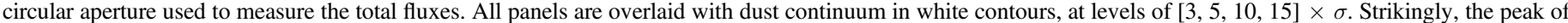

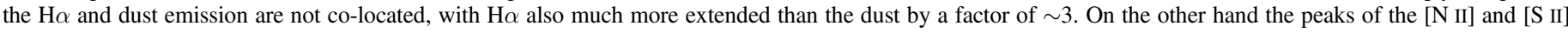

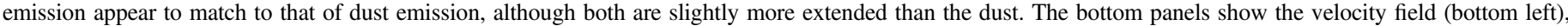

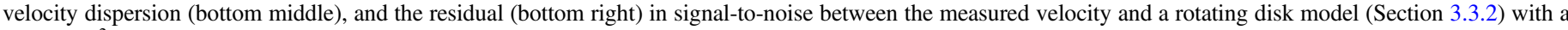

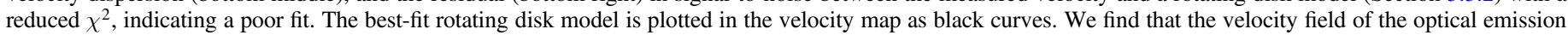
lines is not consistent with the orderly rotating disk.

[N II]-to-H $\alpha$ ratio in the central regions could indicate higher gas-phase metallicity. However, the detected X-ray emission toward ALESS67.1 could also suggest that the elevated ratio is caused by the harder radiation field and/or shocks from AGNs. Deeper observations with detections including [O III] and $\mathrm{H} \beta$ should help distinguish between these alternatives.

Finally, the 2D velocity map shown in Figure 3 displays a velocity gradient in $\mathrm{H} \alpha$ kinematics, with a peak-to-peak velocity difference of $750 \pm 220 \mathrm{~km} \mathrm{~s}^{-1}$. Given the integrated velocity dispersion of $280 \pm 40 \mathrm{~km} \mathrm{~s}^{-1}$ (Section 3.3.1), ALESS67.1 would be considered rotation-dominated based on the criterion of $\left(v_{\max }-v_{\min }\right) / 2 \sigma_{\text {int }}=0.4$, which has been used in some works to roughly differentiate an orderly rotating disk and a merger (e.g., Förster Schreiber et al. 2009). However, the availability of the $2 \mathrm{D}$ velocity map allows us to conduct detailed kinematic modeling to more reliably differentiate rotating systems from mergers.

We start by modeling the velocity field assuming a rotating disc. We adopt the simplest function for the rotational curve, the arctan function (Courteau 1997), with the 1D form of

$$
v(r)=v_{0}+\frac{2}{\pi} v_{\text {asym }} \arctan \left(\frac{r-r_{0}}{r_{t}}\right)
$$

where $v_{0}$ is the systemic velocity, which in our case is zero as the velocity field is referenced at the systematic redshift, $v_{\text {asym }}$ is the asymptotic velocity, $r_{0}$ is the central position, and $r_{t}$ is the transition radius between the rising and flat part of the rotational curve. The arctan function has been found to have the flexibility to reasonably describe $z \gtrsim 1$ rotating galaxies (e.g., Miller et al. 2011; Swinbank et al. 2012). Based on Appendix A of Begeman (1989) the 1D rotational curve is projected to $2 \mathrm{D}$ via

$$
v_{p}(x, y)=v(x, y) \sin (i) \frac{-\left(x-x_{0}\right) \sin \phi+\left(y-y_{0}\right) \cos \phi}{\sqrt{\left(x-x_{0}\right)^{2}+\left(y-y_{0}\right)^{2}}}
$$

where $i$ is the inclination angle, the angle between the normal to the plane of the galaxy and the line of sight (i.e., $0^{\circ}$ if face-on and $90^{\circ}$ if edge-on), $x_{0}$ and $y_{0}$ is the central sky position, and $\phi$ is the positional angle (P.A.) of the major axis, defined as the angle taken in the anticlockwise direction between the north direction in the sky and the major axis of the receding half of the galaxy.

We fit the 2D model to the measured data based on maximum likelihood; in particular we run EMCEE, a Markov chain Monte Carlo (MCMC) ensemble sampler (ForemanMackey et al. 2013), to explore the parameter space and derive uncertainties. We limit the parameters to the range that is allowed by the data; in particular, the center position $\left(x_{0} / y_{0}\right)$ and the turnover radius $\left(r_{t}\right)$ must be within the SINFONI FOV and the P.A. lies between $0^{\circ}$ and $180^{\circ}$. Because $v_{\text {asym }}$ and $i$ are essentially degenerate for our data quality we treat $v_{\text {asym }} \sin (i)$ as a single parameter. 


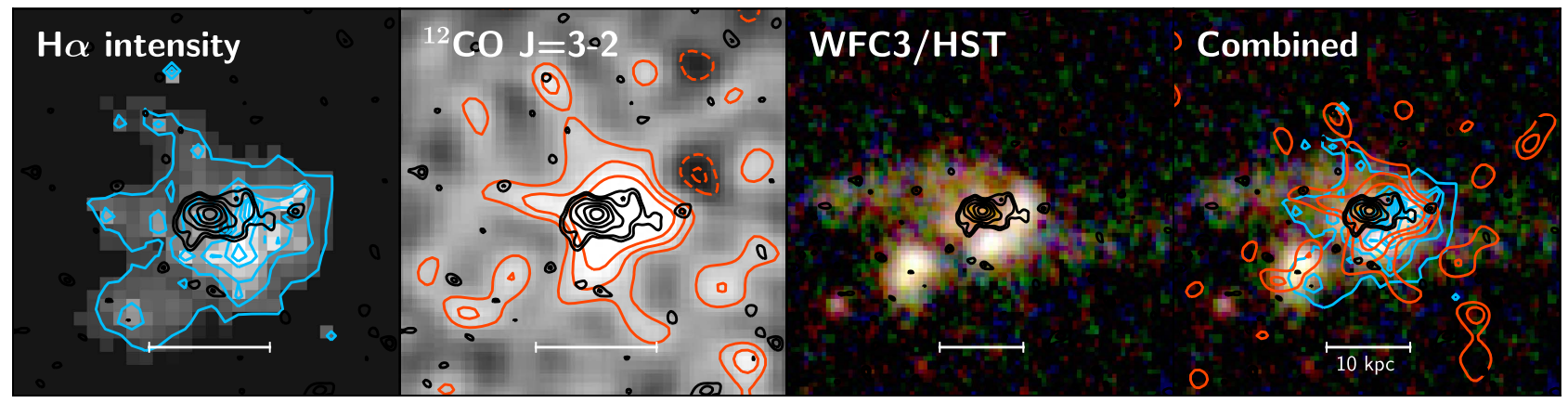

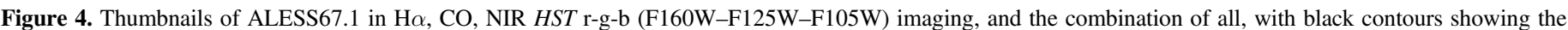

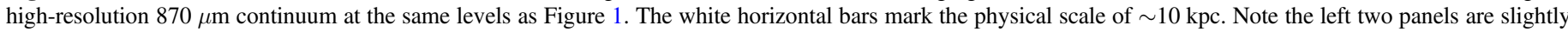

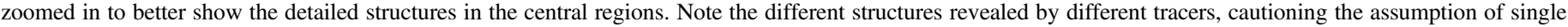
size or morphology when assessing the galaxy properties.

The best-fit model has a $v_{\text {asym }} \sin (i)=290 \pm 70 \mathrm{~km} \mathrm{~s}^{-1}$ and a P.A. of $110^{\circ} \pm 20^{\circ}$, and is plotted over the velocity field in Figure 3, in which the difference between the model and the data is also shown. The reduced $\chi_{\nu}^{2}=14.8$, indicating a relatively poor fit to the data and suggesting ALESS67.1 is not a pure rotating disk.

While modeling the 2D velocity field offers clues as to whether the system is well-described by a simple rotating disk, quantifying the asymmetry of both velocity and velocity dispersion provides a more complete and well-defined view of the kinematics of the system. One well-tested way to measure the asymmetry of the kinematics is to use kinemetry, originally presented by Krajnović et al. (2006) and designed to study local high-S/N stellar kinematic data such as those from the SAURON project (Bacon et al. 2001). It has been further developed into an effective tool to separate disks from mergers (e.g., Shapiro et al. 2008), although at $z>1$ the effectiveness may depend on the interaction stage of the merger (e.g., Hung et al. 2015).

The basic goal of kinemetry analysis is to first decompose the 2D kinematic moment (e.g., velocity and velocity dispersion) maps into a series of concentric ellipses with increasing major axis length, which are defined by the systemic center and positional angle (Krajnović et al. 2006). For each concentric elliptical ring the kinematic moment as a function of the azimuthal angle is then extracted and further decomposed into the Fourier series, which can be described as

$$
K(r, \psi)=A_{0}(r)+\sum_{n=1}^{N} A_{n}(r) \sin (n \psi)+B_{n}(r) \cos (n \psi)
$$

where $r$ is the length of the semimajor axis in each elliptical ring, $\psi$ is the azimuthal angel, and $A_{n}$ and $B_{n}$ are $n$ th-order coefficients. This equation can be shortened to

$$
K(r, \psi)=A_{0}(r)+\sum_{n=1}^{N} k_{n}(r) \cos \left\{n\left[\psi-\phi_{n}(r)\right]\right\}
$$

where $k_{n}=\sqrt{A_{n}^{2}+B_{n}^{2}}$ and $\phi_{n}=\arctan \left(A_{n} / B_{n}\right)$.

In the case of an ideal rotating disk, the kinemetry of the velocity and velocity dispersion field would be dominated by $B_{1}$ and $A_{0}$ coefficients, respectively. Any perturbation from an ideal disk would manifest itself in the higher-order kinemetry coefficients. Therefore the ratios between the high-order and the dominant coefficients in the ideal disk case can be used to quantify the kinematic asymmetry. By using a sample of local galaxies as the training sample Shapiro et al. (2008) proposed a criterion of $K_{\text {asym }}=\left(v_{\text {asym }}^{2}+\sigma_{\text {asym }}^{2}\right)^{1 / 2}=0.5$ to separate rotating disk $\left(K_{\text {asym }}<0.5\right)$ and merger $\left(K_{\text {asym }}>0.5\right)$, where

$$
\begin{aligned}
& v_{\text {asym }}=\left\langle\frac{k_{2, v}+k_{3, v}+k_{4, v}+k_{5, v}}{4 B_{1, v}}\right\rangle_{r}, \\
& \sigma_{\text {asym }}=\left\langle\frac{k_{1, \sigma}+k_{2, \sigma}+k_{3, \sigma}+k_{4, \sigma}+k_{5, \sigma}}{5 B_{1, \sigma}}\right\rangle_{r} .
\end{aligned}
$$

We run the kinemetry code provided by Krajnović et al. (2006) on the velocity and velocity dispersion maps shown in Figure 3. As pointed out by Krajnović et al. (2006) the dominant uncertainty of the kinemetry analyses is the choice of the center position. We therefore adopt the best-fit position based on the previous rotational curve modeling but perturb it within the error obtained from the MCMC analyses. We find a median $v_{\text {asym }}=0.15 \pm 0.01$ and a median $\sigma_{\text {asym }}=0.48 \pm 0.13$, which leads to $K_{\text {asym }}=0.64 \pm 0.15$, suggesting that, judging from the optical line kinematics, ALESS67.1 is a borderline merger (still consistent with a rotating disk given the error) based on the criterion proposed by Shapiro et al. (2008).

\section{Discussion}

We have presented detailed analyses of ALMA and SINFONI observations of the $z=2.12$ SMG ALESS67.1, which provide information on the cold dust continuum, molecular gas, and atomic emission lines predominantly coming from HII regions, all with sub-arcsecond resolution. All of these tracers are resolved in our data and the structures of each tracer are revealed. We compare these structures by overlaying each tracer on top of one another in Figure 4, where we also include the WFC3 imaging from HST, which predominantly show the distribution of unobscured stars. The difference in size and spatial distribution between each tracer, the main finding of this paper, is clear. The availability of all these data means that we can also test some model predictions, as well as attempt to explain recent findings on $z \sim 2$ galaxies regarding the dust attenuation and the deviation of the star formation law. Lastly, the fact that these tracers are not cospatial may have a profound impact on subjects such as galaxy SED modeling, which typically assumes that all components are co-located. In the following we discuss these implications.

\subsection{Kinematics: $\mathrm{CO}$ and $\mathrm{H \alpha}$}

Measuring the kinematics of a galaxy provides an insightful view on its formation and evolution history. For SMGs in 


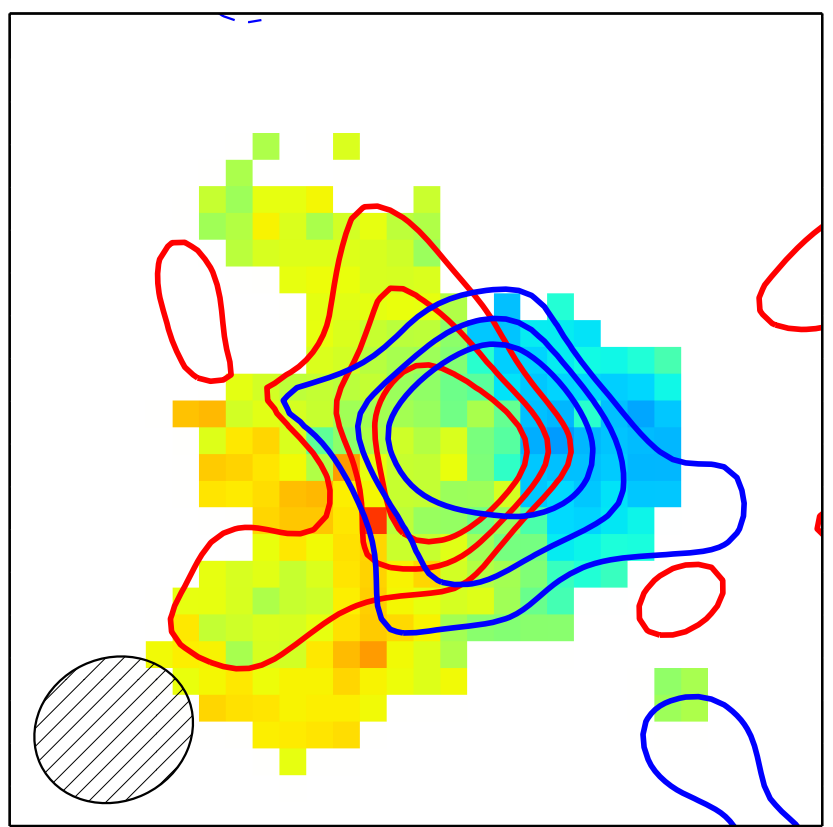

Figure 5. Comparison between velocity of the optical lines derived from SINFONI with that of CO from ALMA overlaid as contours. The approaching side is shown in blue and receding side in red. The synthesized beams are plotted at the bottom-left corner, which has a FWHM of $\sim 0$ ! 7 in major axis. The contours have levels at $[-3,-2,2,3,4] \times \sigma$, in which the positive and negative values are plotted as solid and dashed curves, respectively. The mean velocities of the two $\mathrm{CO}$ channels are $\pm 215 \mathrm{~km} \mathrm{~s}^{-1}$, comparable to the opticalline dynamics. We find that the kinematics of both $\mathrm{H}$ II regions (optical strong lines) and molecular gas (CO) are in broad agreement.

particular, kinematic measurements have mostly been used to assess the mechanisms that drive the enhancement of star formation (e.g., Bouché et al. 2007) and to investigate their evolutionary link to local massive ellipticals (e.g., Swinbank et al. 2006).

However, their high dust obscuration and low surface density means that it is difficult to obtain $\sim \mathrm{kpc}$ resolution optical emission line observations, which require AO for $z \sim 2$ SMGs and are essential to complement the kinematics measured using molecular gas made with ALMA. Consequently there are still only a handful of SMGs that have AO-aided strong optical line data (e.g., Menéndez-Delmestre et al. 2013; Olivares et al. 2016). Together with the technical difficulty of obtaining subarcsecond resolution data for (sub)millimeter molecular lines, in particular low-J CO, most studies have relied on single tracers to assess and compare the kinematics, assuming that different tracers behave similarly. With sub-arcsecond resolution data in hand for both ${ }^{12} \mathrm{CO}(J=3-2)$ and $\mathrm{H} \alpha$ we are in a position to compare these tracers in kinematics.

Our ${ }^{12} \mathrm{CO}(J=3-2)$ detection does not have high enough $\mathrm{S} / \mathrm{N}$ to produce $2 \mathrm{D}$ kinematic maps like $\mathrm{H} \alpha$; we therefore taper the CO map, divide the line in half, and show the averaged maps in both the receding and approaching sides. We overlay the results on the $2 \mathrm{D}$ velocity map obtained from SINFONI in Figure 5.

As revealed in Figure 5, in the outskirts the extended $\mathrm{CO}$ emission appears to follow the bulk rotating motion of the optical emission lines, in particular in the south-east filament. This filament links to a second stellar component revealed in the HST imaging (Figure 4) and could suggest that this second stellar component along with the connecting gas stream was falling toward the dynamical center close to the $870 \mu \mathrm{m}$ continuum. The peak of the blueshifted part of the $\mathrm{CO}$ line agrees with that of the redshifted part; however, higher $\mathrm{S} / \mathrm{N}$ $\mathrm{CO}$ observations are needed to reveal the detailed kinematics in the central regions. In general we find that the kinematics of both $\mathrm{H}$ II regions as traced by the optical emission lines and molecular gas as traced by ${ }^{12} \mathrm{CO}(J=3-2)$ are in broad agreement.

\section{2. $\alpha_{C O}$}

The interstellar medium of the dense environments in which rapid star formation occurs is dominated by molecular hydrogen, $\mathrm{H}_{2}$. As a result $\mathrm{H}_{2}$ plays a central role in the formation and evolution of galaxies (Kennicutt \& Evans 2012). While cold $\mathrm{H}_{2}$ is not directly observable in emission, ${ }^{12} \mathrm{CO}$ has been widely used to trace total molecular gas, with a standard conversion between ${ }^{12} \mathrm{CO}$ luminosity and the total molecular gas mass, $M_{\mathrm{mol}}=\alpha_{\mathrm{CO}} L_{\mathrm{CO}(1-0)}^{\prime}$, in which $\alpha_{\mathrm{CO}}$ is the CO-to- $\mathrm{H}_{2}$ conversion factor. For extragalactic sources, the conversion factor depends on the properties of the galactic environments such as gas density and metallicity (Bolatto et al. 2013), resulting in a factor of $\sim 6$ variation between local mergers and local spiral galaxies (Downes \& Solomon 1998).

Previous studies have attempted to quantify $\alpha_{\mathrm{CO}}$ for SMGs; however, subject to a lack of constraints on dust mass and/or the size and dynamics of the $\mathrm{CO}$ emission, the results were generally not conclusive (e.g., Bothwell et al. 2013), though on average SMGs were found to have a low $\alpha_{\mathrm{CO}}$ (Danielson et al. 2011). With all the necessary measurements in hand for the case of a merger we can quantify and test the link between the merger and $\alpha_{\mathrm{CO}}$.

To measure $\alpha_{\mathrm{CO}}$ we first adopt a dynamical method, in which the gas mass is derived by subtracting stellar and darkmatter mass from the dynamical mass (i.e., $M_{\text {dyn }}\left(r \leqslant r_{e}\right)=$ $0.5 \times\left(M_{\mathrm{star}}+M_{\mathrm{gas}}\right)+M_{\mathrm{dark}}\left(r \leqslant r_{e}\right)$, where $r_{e}$ represents the half-light radius and we assume the gas and stars have the same $r_{e}$ given this is what we find (Figure 6). Within the half-light radius the dark matter contribution $\left(M_{\mathrm{dark}}\right)$ is estimated to be $10 \%-20 \%$ of the dynamical mass in $z \sim 2$ star-forming galaxies (e.g., Genzel et al. 2017) so we adopt $15 \%$. Since we find that ALESS67.1 is rotation-dominated based on the optical emission lines Section 3.3.2, the dynamical masses can be estimated using Newtonian dynamics assuming a point mass, $v_{c}\left(r_{e}\right)=\sqrt{G M_{\mathrm{dyn}}\left(<r_{e}\right) / r_{e}}$, in which $v_{c}$ is the circular velocity and $G$ is the gravitational constant. Based on the dynamical modeling presented in Section 3.3.2 $v_{c}$ can be described by Equation (1). However in a highly turbulent environment $\left(v_{\text {asym }} / \sigma \lesssim 3\right)$, the rotational velocity is significantly reduced due to turbulent pressure effects and needs to be corrected, with a form of $v_{c}^{2}\left(r_{e}\right)=v^{2}\left(r_{e}\right)+3.36 \times \sigma^{2}$ (Equation (11) in Burkert et al. 2010 assuming exponential surface density distribution). This correction assumes constant velocity dispersion $(\sigma)$ over the spatial extent, which is supported by our data (Figure 3). We derive a median $\sigma$ of $154 \pm 5 \mathrm{~km} \mathrm{~s}^{-1}$ with a bootstrapped error. By adopting an averaged inclination $\left\langle\sin ^{2}(i)\right\rangle=2 / 3$ (Tacconi et al. 2008) and a half-light radius of $r_{1 / 2, \mathrm{H} \alpha}=6.6 \pm 0.9 \mathrm{kpc}$ we derive $v_{c}\left(r_{e}\right)=380 \pm 40 \mathrm{~km} \mathrm{~s}^{-1}$ giving a dynamical mass of $M_{\mathrm{dyn}}\left(r \leqslant r_{e}\right)=(2.2 \pm 0.6) \times$ $10^{11} M_{\odot}$. The stellar mass is estimated to be $M_{\text {star }}=2 \times 10^{11} M_{\odot}$ (Simpson et al. 2014; da Cunha et al. 2015). Hence we estimate a gas mass $M_{\text {gas }}=(1.8 \pm 1.0) \times 10^{11} M_{\odot}$. Given 


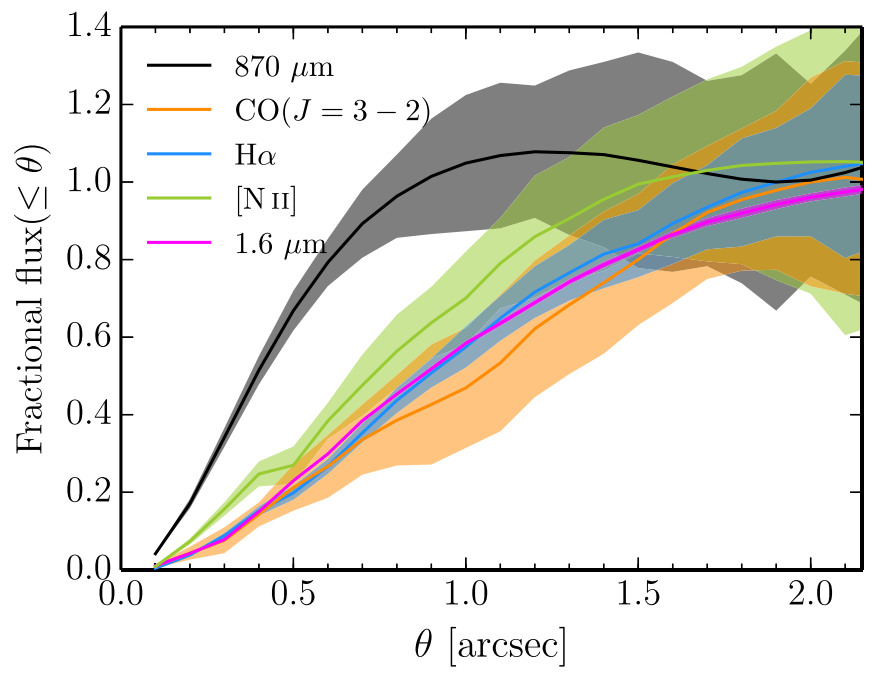

Figure 6. Curve-of-growth diagram with all the tracers plotted. All the maps are convolved with the $\mathrm{CO}$ beam to make the spatial resolution comparable. Note the size difference between $870 \mu \mathrm{m}$ and the rest of the tracers, especially CO. Our results caution against assumptions in any model or analysis that adopt a single geometry for all the tracers in SMGs.

$L_{\mathrm{CO}(1-0)}^{\prime}=(9.9 \pm 1.8) \times 10^{10} \mathrm{~K} \mathrm{~km} \mathrm{~s}^{-1} \mathrm{pc}^{2}$ (Huynh et al. 2017), we derive $\alpha_{\mathrm{CO}}=1.8 \pm 1.1$.

We can also check our $\alpha_{\mathrm{CO}}$ estimate by using the gas-to-dust ratio method. The idea is that by estimating the gas-to-dust mass ratio, $\delta_{\mathrm{GDR}}$, in a galaxy with measured molecular gas and dust masses, the conversion factor $\alpha_{\mathrm{CO}}$ can be derived as $\alpha_{\mathrm{CO}}=\delta_{\mathrm{GDR}}\left(\mu_{0}\right) M_{\mathrm{dust}} / L_{\mathrm{CO}(1-0)}^{\prime}$ assuming that molecular gas dominates the gas masses, and $\delta_{\mathrm{GDR}}$ can be related to gas phase metallicity $\left(\mu_{0}\right.$; Leroy et al. 2011). Assuming negligible AGN contribution to the $[\mathrm{N} \mathrm{II}] / \mathrm{H} \alpha$ ratio, we derive a metallicity of 12 $+\log (\mathrm{O} / \mathrm{H})=8.8 \pm 0.1$ in the central region, and $8.6 \pm 0.1$ over the entire galaxy, by adopting the $N 2 \equiv \log _{10}([\mathrm{~N}$ II $] \lambda 6583 / \mathrm{H} \alpha)$ empirical calibration provided by Pettini \& Pagel (2004) $(12+$ $\left.\log _{10}(\mathrm{O} / \mathrm{H})=8.90+0.57 \times N 2\right)$. By using a circular aperture on the $\mathrm{CO}(J=3-2)$ cube centered at the $870 \mu \mathrm{m}$ continuum peak with a deconvolved radius matching to the dusty region $(\leqslant 0$ !" 4$)$, we estimate $L_{\mathrm{CO}(1-0)}^{\prime}=(4.0 \pm 1.9) \times 10^{10} \quad \mathrm{~K} \mathrm{~km} \mathrm{~s}^{-1} \mathrm{pc}^{2}$. Based on the metallicity measurement and the best-fit linear function provided by Leroy et al. (2011), we compute $\delta_{\mathrm{GDR}}$ of $78 \pm 6$ in the central region. By adopting the dust mass of ALESS67.1 derived by Swinbank et al. (2014), we calculate a gas mass of $(5.5 \pm 0.6) \times 10^{10} M_{\odot}$, and therefore $\alpha_{\mathrm{CO}, \mathrm{GDR}}=$ $1.4 \pm 0.7$ in the central dusty regions, in good agreement with the $\alpha_{\mathrm{CO}}$ estimated using the dynamical method. On the other hand, it is also possible that the apparent size difference between dust and $\mathrm{CO}$ is caused by observational bias due to the different optical depth probed by $\mathrm{CO}$ and $870 \mu \mathrm{m}$ continuum. In such a case $\mathrm{CO}$ and dust are still well-mixed in the entire galaxy and the total CO luminosity needs to be adopted for the gas-to-dust ratio method. If we do so and adopt global integrated values for all relevant measurements, we obtain a lower $\alpha_{\mathrm{CO}}$ of $0.5 \pm 0.3$, consistent with the results derived using other methods. The results of the gas-to-dust ratio method should be treated as lower limits as the AGN contribution could lower the gas-phase metallicity and hence suggest higher gas masses.

Despite the uncertainty, our result is consistent with that of another strongly lensed SMG, SMMJ2135-0102 (Cosmic Eyelash; Danielson et al. 2011, 2013; Thomson et al. 2015), and Arp 220 (Scoville et al. 1997), a local merger that has been used to compare with $z \sim 2$ SMGs, with both having $\alpha_{\mathrm{CO}} \sim 1$. Confirming the results from other analyses, our results on $\alpha_{\mathrm{CO}}$ suggest a consistent scenario that ALESS67.1 is undergoing a merger.

\subsection{Sizes and Morphology}

Recent SMG studies of kpc-scale dust distributions using ALMA (sub)millimeter observations have found, almost unequivocally, compact sizes with an average half-light radius of 1-2 kpc (e.g., Ikarashi et al. 2015; Simpson et al. 2015; Hodge et al. 2016; Spilker et al. 2016). Above an IR luminosity of $\sim 3 \times 10^{12} L_{\mathrm{IR}}\left(\mathrm{SFR} \sim 300 M_{\odot} \mathrm{yr}^{-1}\right)$, the dust sizes do not appear to depend on $L_{\mathrm{IR}}$ and redshift $(z \sim 1-6)$, and they are on average a factor of 2-3 smaller than the NIR continuum revealed by the HST (Hodge et al. 2016).

We have presented analyses and measured sizes and spatial distributions of CO, optical emission lines, $870 \mu \mathrm{m}$ and NIR continuum for ALESS67.1. We summarize part of the measurements in Figure 6 in a curve-of-growth style to emphasize the size difference between each tracer. All maps are convolved with the ${ }^{12} \mathrm{CO}$ beam to make the spatial resolution comparable.

Besides confirming the size contrast between $870 \mu \mathrm{m}$ and $1.6 \mu \mathrm{m}$ continuum, we also find significantly larger sizes for all other tracers with respect to the $870 \mu \mathrm{m}$ dust continuum. Perhaps the most striking of all is the factor of $5.2 \pm 0.8$ difference in half-light radius between the FIR/submillimeter dust continuum and the ${ }^{12} \mathrm{CO}(J=3-2)$ gas, which is normally found to agree within a factor of two in nearby star-forming galaxies (e.g., Sandstrom et al. 2013), as well as local mergers such as Arp220 (Scoville et al. 1991), NGC 6240 (Iono et al. 2007), and NGC 3256 (Sakamoto et al. 2006).

The measured ${ }^{12} \mathrm{CO}(J=3-2)$ size of $\operatorname{ALESS67.1}$ is consistent with that of ${ }^{12} \mathrm{CO}(2-1)$ from the $z=4.055$ SMG GN20 (Hodge et al. 2012), as well as that of ${ }^{12} \mathrm{CO}(1-0)$ in a sample of four SMGs presented in Ivison et al. (2011) and the $z=3.408$ SMG SMMJ13120+4242 (Riechers et al. 2011). However, it is significantly larger than the ${ }^{12} \mathrm{CO}$ sizes reported by Engel et al. (2010), in which they claimed an average halflight radius of $2.5 \pm 1.3 \mathrm{kpc}$ for ${ }^{12} \mathrm{CO}$ lines with upper $J \leqslant 6$. The difference could be a reflection of true scatter in ${ }^{12} \mathrm{CO}$ sizes, which is a factor of $\sim 8$ in local mergers (Ueda et al. 2014) or, as claimed by Ivison et al. (2011) that low- $J$ lines are more spatially extended than high- $J$ ones, or both.

On the other hand, in terms of spatial extent and surface brightness, we find that $\mathrm{H} \alpha$ follows a similar distribution as the stellar components traced by the NIR emission. When comparing the $\mathrm{H} \alpha / \mathrm{NIR}$ continuum to ${ }^{12} \mathrm{CO}$ we find similar sizes.

When comparing to the measurements in the literature, the NIR size of ALESS67.1, which is $6.4 \pm 0.5 \mathrm{kpc}$ using the curve-of-growth method (Figure 6), lies within the average size of the ALESS parent sample (Chen et al. 2015) and, given the stellar mass estimates, is consistent with the size census on mass-selected samples of star-forming galaxies at $z \sim 2$ (van der Wel et al. 2014). The NIR size of ALESS67.1 is, however, significantly larger than that of $500 \mu \mathrm{m}$ selected DSFGs reported by Calanog et al. (2014), in which they attribute the difference to the selection bias caused by the strong gravitational lensing in their sample.

The $\mathrm{H} \alpha$ size of ALESS67.1 lies at the higher end but still consistent with respect to other SMGs reported in 
Alaghband-Zadeh et al. (2012) (average $3.7 \pm 0.8 \mathrm{kpc}$ ) and main-sequence SFGs (Förster Schreiber et al. 2009), but significantly larger than the $\mathrm{H} \alpha$ emitters (HAEs) at similar reshifts (Molina et al. 2017). The difference between ALESS67.1 and HAEs, apart from small samples, could be caused by the fact that the HAE sample presented in Molina et al. is on average a factor of $>3$ less massive than ALESS67.1.

Finally, the geometrical discrepancy among different tracers, which is also seen in other studies of high- $z$ galaxies (e.g., Spilker et al. 2015; Decarli et al. 2016; Ginolfi et al. 2017; Koprowski et al. 2016), may have some impact on theoretical modeling. As already discussed in Simpson et al. (2017), geometrical differences in SMGs between dust and UV-to-NIR emissions leads to drastically different estimations for dust extinction, with Simpson et al. deriving an $A_{\mathrm{V}}=540_{-40}^{+80}$ using a hydrogen column density method based on direct measurements of dust column density, in contrast to just $A_{\mathrm{V}} \sim 1-3$ based on SED modeling of the detectable optical emissions either using simple dust screen modeling (Simpson et al. 2014) or an energy-balance approach (da Cunha et al. 2015). For SMGs, because of this geometrical discrepancy, it may be more sensible to model optical-to-NIR SEDs and FIR/submillmeter/ radio SEDs separately.

In the next two sections we discuss the impact of this geometrical discrepancy on the IRX- $\beta$ and Schmidt-Kennicutt relationships.

\section{4. $I R X-\beta$}

The relationship between the ratio of IR and UV luminosity at $1600 \AA$ (IRX) and the UV spectral slope at $1600 \AA$ ( $\beta$ ) offers a potential route to estimate the total SFR when only the restframe UV observations are available. This IRX $-\beta$ relationship has therefore been widely used to estimate the total star formation rate density for $\mathrm{UV}$-selected populations at $z>3$ and up to the epoch of reionization (e.g., Bouwens et al. 2015).

Given its fundamental implications for the measurements of SFR density at high redshifts, the IRX $-\beta$ relationship and its deviation has been extensively studied both in the local universe and at high redshifts (e.g., Meurer et al. 1999; Kong et al. 2004; Buat et al. 2005; Howell et al. 2010; Overzier et al. 2011; Reddy et al. 2012; Takeuchi et al. 2012; Casey et al. 2014b; To et al. 2014). Among the many factors that affect the IRX $-\beta$ relationship, such as star formation history and internal attenuation curve, the geometrical effect has been proposed to explain the deviation seen in samples of ULIRGs, both in the local universe (Howell et al. 2010) and at $z \sim 2$ (Casey et al. $2014 b$ ), in a sense that the different or completely decoupled geometry between dust and UV could explain the increase of the IRX at a fix $\beta$. Recent theoretical models have also confirmed this hypothesis (e.g., Narayanan et al. 2017; Popping et al. 2017). With $\sim \mathrm{kpc}$ resolution data in rest-frame UV, optical, and FIR, we are set to examine this proposal.

We first compute the UV luminosity and spectral slope at $1600 \AA$ A. ALESS67.1 is covered by CANDELS imaging (Figure 4) as well as 3D-HST grism spectroscopy (Brammer et al. 2012), and the multi-wavelength photometry and restframe UV flues at 1400, 1700, 2200, 2700, and $2800 \AA$ are provided in Skelton et al. (2014). We adopt the values in the 3D-HST catalog and derive the spectral slope by fitting the data with a functional form of $F(\lambda)=A \lambda^{\beta}$. We then use this bestfit function to compute the rest-frame UV flux at $1600 \AA$ in units of erg s${ }^{-1} \mathrm{~cm}^{2} \AA^{-1}$ and calculate the UV luminosity using $L_{\mathrm{UV}}=4 \pi D_{\mathrm{L}}^{2} F(1600) \lambda_{1600} /(1+z)$, in which $D_{\mathrm{L}}$ is the luminosity distance at redshift $z$. The IR luminosity is adopted from Swinbank et al. (2014), which uses deblended Herschel data. The results are plotted in Figure 7, in which we also show the composite image of dust, stars, and UV emission.

Consistent with the trend found by Casey et al. (2014b), with an $L_{\mathrm{IR}}$ of $10^{12.7} L_{\odot}$ ALESS67.1 lies significantly above the relationships found by other studies for less IR-luminous galaxies. As shown in the right panel of Figure 7 the completely decoupled geometry between rest-frame $280 \mu \mathrm{m}$ and rest-frame $1400 \AA$ emission confirms the hypothesis that the deviation from the IRX $-\beta$ relationship is caused, at least partially, by the different distributions between dust and UV. For the individual UV-emitting regions, on the other hand, our ALMA data are not sensitive enough to put meaningful upper limits on whether or not they lie on the relationships (left panel in Figure 7). This is the same with the dusty regions, where we do not have meaningful constraints on either UV luminosity or slope.

\subsection{The Schmidt-Kennicutt Relationship}

The relationship between the surface density of star formation rate and that of gas has been studied back to the seminal work of Schmidt (1959) and Kennicutt (1989). Considering galaxies as a whole and assuming a single $\alpha_{\mathrm{CO}}$, it was later claimed in observations that this tight correlation, the Schmidt-Kennicutt relationship, with a scatter about a factor of two, holds valid in local star-forming galaxies over six orders of magnitude in SFR surface density (Kennicutt 1998b).

However, studies of local interacting mergers have shown a significantly lower $\alpha_{\mathrm{CO}}\left(\alpha_{\mathrm{CO}} \sim 1\right.$; e.g., Scoville et al. 1997; Downes \& Solomon 1998), which could also be true at high redshifts based on our analyses of ALESS67.1 in Section 4.2. By adopting the lower $\alpha_{\mathrm{CO}}$ for (U)LIRGs, together with observations of $z \sim 2$ star-forming galaxies and SMGs, it has been claimed that the Schmidt-Kennicutt relationship becomes bimodal, in which IR-luminous galaxies have significantly higher SFR surface density at a fixed gas surface density, with a typical gas consumption time $\left(\Sigma_{\text {gas }} / \Sigma_{\mathrm{SFR}}\right)$ of $\sim 100 \mathrm{Myr}$, in contrast to $\sim 1$ Gyr for normal SFGs (e.g., Daddi et al. 2010; Genzel et al. 2010). Recent spatially resolved observations of SMGs on both star-forming regions as traced by dust or radio emissions and $\mathrm{CO}$ have found an even shorter gas consumption time of $10 \mathrm{Myr}$ (Bothwell et al. 2010; Hodge et al. 2015). With measured luminosities and sizes on FIR, CO, and $\mathrm{H} \alpha$ we are in a position to investigate the Schmidt-Kennicutt relationship on a $z \sim 2$ merger.

We first compute the global surface density of SFR and $\mathrm{H}_{2}$ for ALESS67.1, by adopting the measured sizes of each tracer and the conversion between $L_{\mathrm{IR}}$ and $L_{\mathrm{H} \alpha}$ to SFR from Kennicutt (1998a). We assume a Chabrier initial mass function (Chabrier 2003) and adopt 0.8 for $\alpha_{\mathrm{CO}}$ in order to make comparisons with other studies. The results are plotted in Figure 8, in which we also include literature values from local and high-redshift SFGs and SMGs. We only include measurements obtained from observations that spatially resolve both the star-forming regions and $\mathrm{CO}$, which is the condition of our data.

Our measurements agree with GN20, an SMG at $z \sim 4$, as well as some SMGs in the sample of Bothwell et al. (2010), suggesting that, when looking at the galaxy as a whole, some SMGs have significantly shorter gas consumption times even 

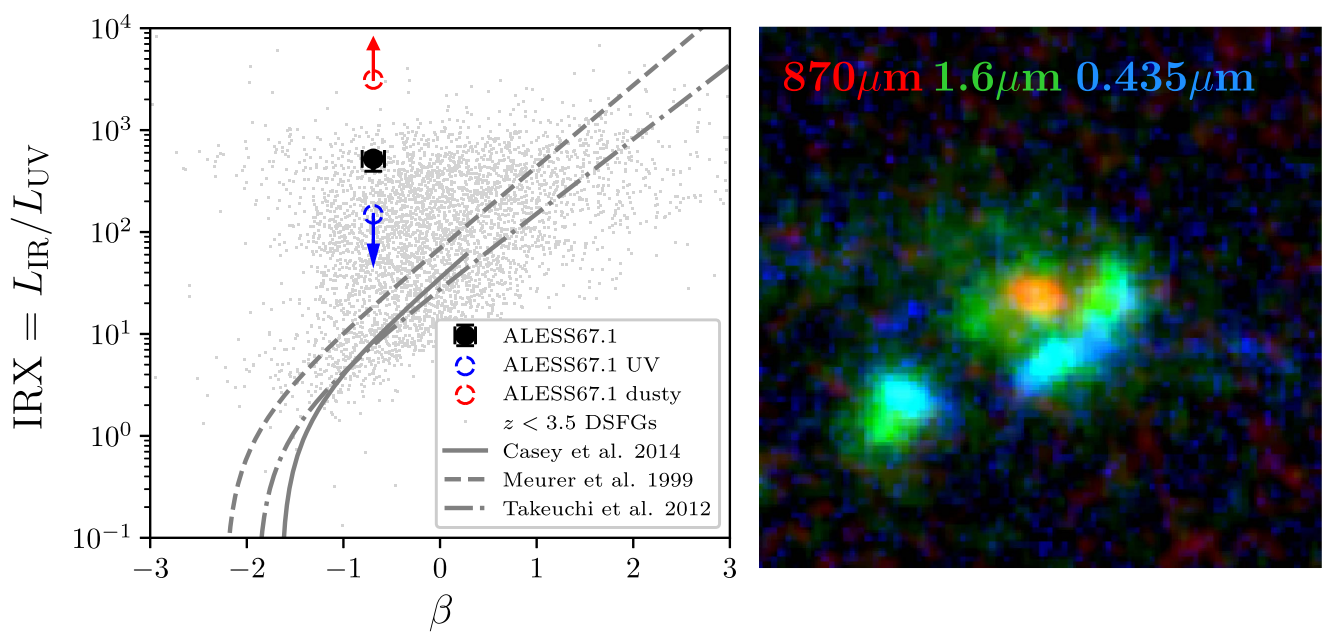

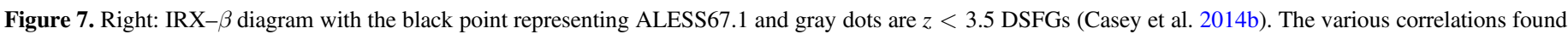

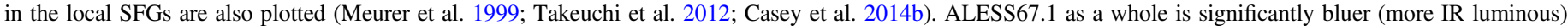

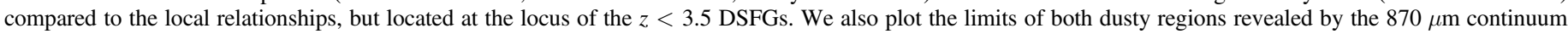

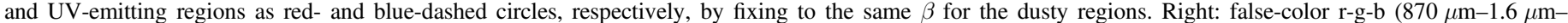

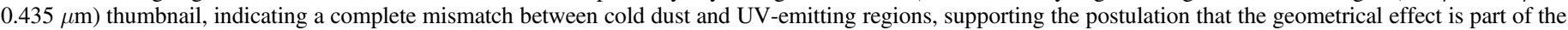
reason for the deviation of DSFGs on the local IRX- $\beta$ correlations.

compared to the local mergers. However, because of the dramatic size difference between the dusty star-forming regions, which dominate the SFR surface density, and $\mathrm{CO}$, the comparison of $\Sigma_{\mathrm{H}_{2}}$ and $\Sigma_{\mathrm{SFR}}$ on the global scale is nonphysical, meaning the two parameters come from regions that are largely unrelated, and therefore the deviation from the typical relationship is expected. To make proper interpretations it is necessary to measure both parameters in resolved regions.

Motivated by the spatial distribution of the $870 \mu \mathrm{m}$ continuum, ${ }^{12} \mathrm{CO}(J=3-2)$, and $\mathrm{H} \alpha$, we construct concentric rings with a width of $0.15(\sim 4 \mathrm{kpc})$ for each ring and centered at the peak of the $870 \mu \mathrm{m}$ continuum. The choice of width is determined by the spatial resolution of $\mathrm{CO}$. We convolve the $870 \mu \mathrm{m}$ continuum and $\mathrm{H} \alpha$ with a $\mathrm{CO}$ beam to match the maps in spatial resolution. We then measure $\Sigma_{\mathrm{H}_{2}}$ and $\Sigma_{\mathrm{SFR}}$ for each concentric ring and plot the results in the right panel of Figure 8, color coded based on their radial distances.

Unlike the global values which make ALESS67.1 almost an outlier compared to the local IR-selected galaxies, the measurements in each sub-region lie in the similar locus occupied by previous measurements. In particular, within one galaxy we obtain a similar bimodal distribution as seen between normal SFGs and mergers. The transition, which is at $\sim 5 \mathrm{kpc}(\sim 0$ " 5$)$ in this case, occurs when there is a lack of detection at $870 \mu \mathrm{m}$, leaving $\Sigma_{\mathrm{SFR}}$ derived solely from $\mathrm{H} \alpha$. We should stress, however, that these values are derived under the assumption of $\alpha_{\mathrm{CO}}=0.8$, and $\mathrm{H} \alpha$ is not corrected for dust extinction, if any. If the sub-regions in the outskirts had a Galactic $\alpha_{\mathrm{CO}}$ instead, the derived quantities would shift to higher $\Sigma_{\mathrm{H}_{2}}$ by $\sim 0.7$ dex, which would make these regions significantly more gas-rich, or significantly inefficient in star formation. This result is mainly the consequence of the much more extended distribution of ${ }^{12} \mathrm{CO}(J=3-2)$ compared to the dust traced by the rest-frame $280 \mu \mathrm{m}$ continuum.

\section{Summary}

We present detailed spatial and dynamical studies of an SMG, ALESS67.1, using sub-arcsecond resolution ALMA, AO-aided SINFONI, and HST data to investigate the properties of cold dust (rest-frame $280 \mu \mathrm{m}$ continuum), ${ }^{12} \mathrm{CO}(J=3-2)$, optical emission lines ( $\mathrm{H} \alpha,[\mathrm{N}$ II], [S II]), and stellar continuum (HST imaging). ALESS67.1 has a submillmeter flux $\left(S_{850} \sim 4\right.$ $\left.\mathrm{mJy}, \mathrm{SFR} \sim 500 M_{\odot} \mathrm{yr}^{-1}\right)$ and redshift $(z=2.12)$ which make it typical among the general SMG population covered by single-dish submillimeter surveys. Our findings are summarized as follows.

1. By conducting detail dynamical analyses on optical lines and CO, we find that ALESS67.1 is not consistent with an isolated, pure rotating disk. This is supported by the stellar morphology revealed in the HST imaging, showing tidal features typically seen in major mergers in the local universe. Considering the compact dusty star formation we conclude that ALESS67.1 is likely a final, coalescentstage merger.

2. We find that the kinematics of $\mathrm{H} \alpha$ and ${ }^{12} \mathrm{CO}(J=3-2)$ are in broad agreement, although higher $\mathrm{S} / \mathrm{N} \mathrm{CO}$ observations are needed to make a detailed comparison in the central regions.

3. All tracers are resolved at the spatial resolution of our observations, and we have measured the half-light radius for each of them, finding $1.2 \pm 0.1 \mathrm{kpc}$ (circularized) for the $870 \mu \mathrm{m}$ (rest-frame $280 \mu \mathrm{m}$ ) continuum, $6.5 \pm 0.9 \mathrm{kpc}$ for ${ }^{12} \mathrm{CO}(J=3-2), 6.6 \pm 0.9 \mathrm{kpc}$ for $\mathrm{H} \alpha, 5.1 \pm 1.7 \mathrm{kpc}$ for [N II], $5.1 \pm 2.1 \mathrm{kpc}$ for [S II], and $6.4 \pm 0.5 \mathrm{kpc}$ for the stellar continuum at $1.6 \mu \mathrm{m}$ (rest-frame $\sim 5000 \AA$ ). We therefore find that the dust continuum has a factor of 4-6 smaller size than that of the strong optical emission lines, NIR continuum, and ${ }^{12} \mathrm{CO}(J=3-2)$, and it significantly offsets from the peaks of $\mathrm{H} \alpha$ and the rest-frame optical stellar continuum. While the ${ }^{12} \mathrm{CO}(J=3-2)$ size is consistent with $\mathrm{H} \alpha$ and the stellar continuum, the peak emissions encloses both obscured (rest-frame FIR continuum) and unobscured $(\mathrm{H} \alpha)$ star formation, supporting that ${ }^{12} \mathrm{CO}(J=3-2)$ traces star-forming gas.

4. Using the dynamical method we derive a CO-to- $\mathrm{H}_{2}$ conversion factor of $\alpha_{\mathrm{CO}}=1.8 \pm 1.0$, supported by estimates based on the gas-to-dust ratio method. Our results are also consistent with the lensed SMGs 

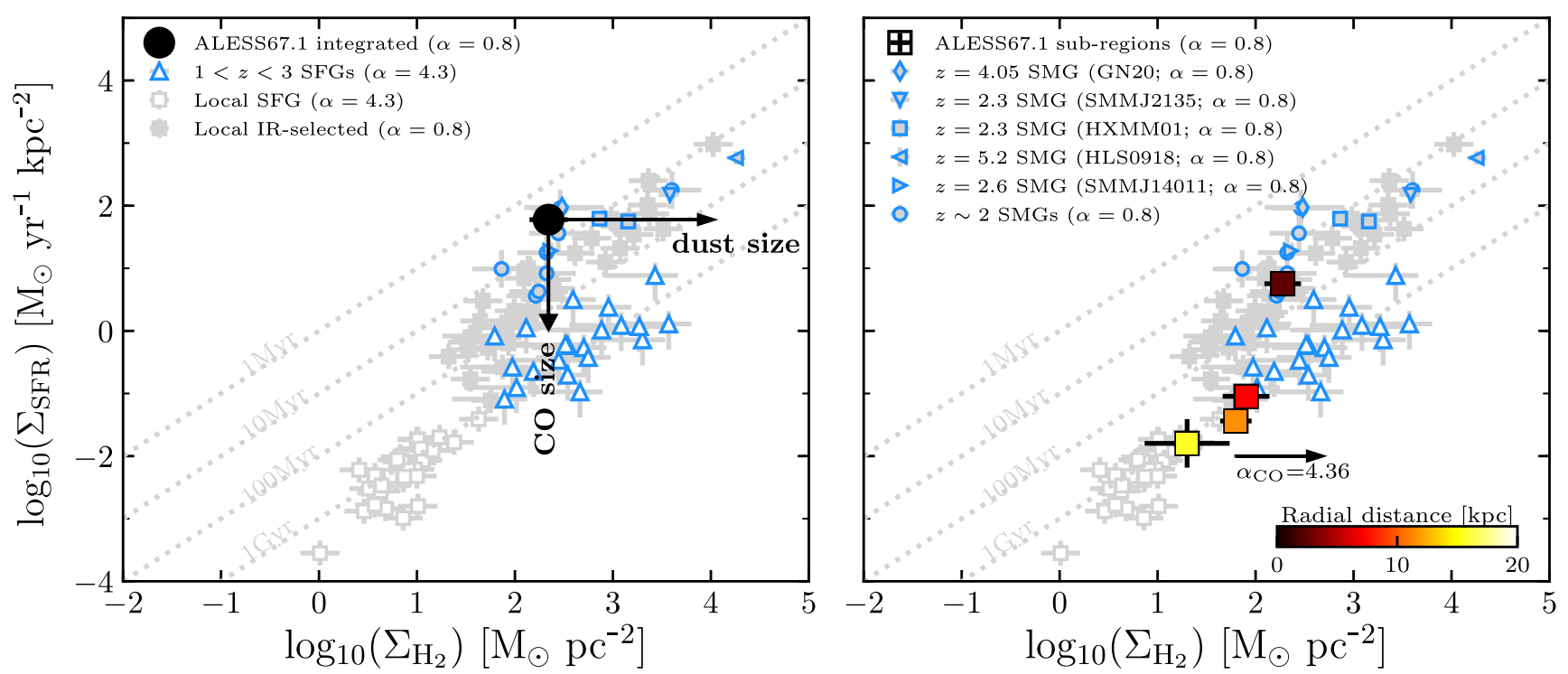

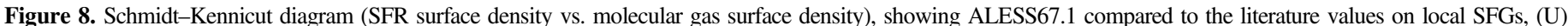

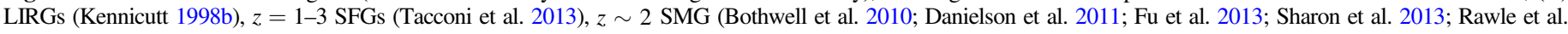

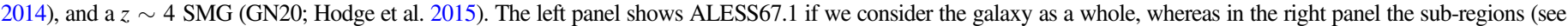

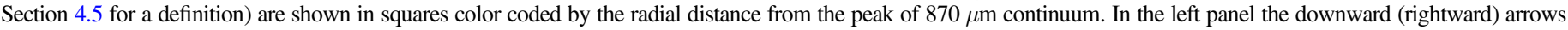

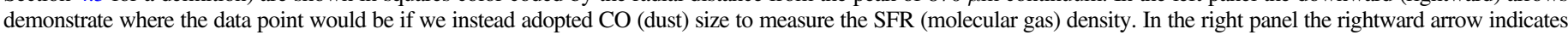

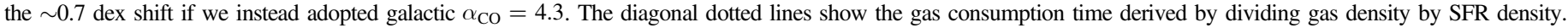

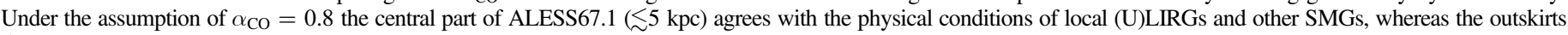
$(\gtrsim 5 \mathrm{kpc}$ ) of the galaxy follow closely with both local and $z \sim 2$ SFGs.

(Danielson et al. 2011; Spilker et al. 2015) and the local merger Arp220 (Scoville et al. 1997), and suggest values of $\alpha_{\mathrm{CO}} \sim 1-2$ are appropriate for high-redshift dusty galaxies.

5. We show that the striking difference in spatial distribution between dust and the UV continuum could be part of what drives ALESS67.1, as well as other dusty starforming galaxies, off the IRX- $\beta$ relationship found locally, which is widely used to attempt to infer dustobscured star formation for $\mathrm{UV} /$ optical-selected galaxies at $z \gtrsim 4$.

6. We demonstrate that, when considering the galaxy as a whole, the compact dusty star formation coupled with extended $\mathrm{CO}$ could be the cause of unusually high efficiency in star formation found recently in some SMGs (Figure 8). However, when looking at the gas density and SFR density in individual sub-regions (with the assumption of $\alpha_{\mathrm{CO}}=0.8$ ), we find them consistent with previous studies, in both the core part of the galaxy $(\lesssim 5 \mathrm{kpc})$ and the outskirts ( $\gtrsim 5 \mathrm{kpc}$ ), although each following a different path: the core shares the same locus as mergers whereas the outskirts lie close to the SFGs selected in the UV/optical, suggesting different star formation efficiencies within one galaxy.

Given the spatial variability of different tracers found within ALESS67.1, our results demonstrate the importance of using high spatial resolution, multi-wavelength data to interpret the properties of SMGs and, more generally, the less IR-luminous $z \gtrsim 2$ star-forming galaxies. In particular, there is growing evidence of a geometrical discrepancy between $\mathrm{CO}$ and dust among galaxies, regardless of them being SMGs or not, either mismatching in spatial distributions (Riechers et al. 2011), or in sizes (Hodge et al. 2015; Spilker et al. 2015; Decarli et al. 2016). For less IR-luminous galaxies, cases of mismatch between dust and UV/optical emissions (Koprowski et al. 2016), as well as very extended CO and dust (Ginolfi et al. 2017), have recently been reported. Given that ALMA is now reaching its full capability, we expect to see more and more such cases (e.g., Tadaki et al. 2017). The physical interpretation of these results, especially the mismatch between $\mathrm{CO}$ and dust, needs to be explored further, both theoretically and observationally. It could be that the rest-frame $280 \mu \mathrm{m}$ continuum still misses a significant amount of cold, optically thin dust, which requires higher surface brightness sensitivity and is better traced by optically thick CO. Deeper ALMA observations in millimeter wavelengths coupled with hydrodynamical simulations implemented with detailed radiative transfer treatment might shed more light on this issue.

We acknowledge the referee for a helpful report that has improved the manuscript. We would like to thank Nick Scoville, Desika Narayanan, and Gergö Popping for discussions. C.-C.C. and I.R.S. acknowledge support from the ERC Advanced Investigator programme DUSTYGAL 321334. C.-C.C. also acknowledges support from the European Southern Observatory through a fellowship program. J.A.H. acknowledges support of the VIDI research programme with project number 639.042.611, which is (partly) financed by the Netherlands Organisation for Scientific Research (NWO). I.R.S. also acknowledges support from a Royal Society/Wolfson Merit Award and STFC through grant number ST/L00075X/1. H.D. acknowledges financial support from the Spanish Ministry of Economy and Competitiveness (MINECO) under the 2014 Ramón y Cajal program MINECO RYC-2014-15686. This research made use of Astropy, a community-developed core Python package for Astronomy (Astropy Collaboration et al. 2013). This research made use of NASA's Astrophysics Data System. This paper makes use of the following ALMA data: ADS/JAO.ALMA \# 2012.1.00307.S and 2013.1.00407.S. ALMA is a partnership of ESO (representing its member states), NSF (USA) and NINS (Japan), together with NRC (Canada) and NSC and ASIAA 
(Taiwan) and KASI (Republic of Korea), in cooperation with the Republic of Chile. The Joint ALMA Observatory is operated by ESO, AUI/NRAO and NAOJ.

\section{ORCID iDs}

\section{Chian-Chou Chen}

(陳建州) (1D https://orcid.org/0000-0002-3805-0789

J. A. Hodge (10 https://orcid.org/0000-0001-6586-8845

Ian Smail (i) https://orcid.org/0000-0003-3037-257X

A. M. Swinbank (1) https://orcid.org/0000-0003-1192-5837

Fabian Walter (iD https://orcid.org/0000-0003-4793-7880

J. M. Simpson (ib https://orcid.org/0000-0002-8521-1995

F. Bertoldi (10 https://orcid.org/0000-0002-1707-1775

H. Dannerbauer (iD https://orcid.org/0000-0001-7147-3575

C. De Breuck (i) https://orcid.org/0000-0002-6637-3315

C. M. Harrison (ib https://orcid.org/0000-0001-8618-4223

R. J. Ivison (ib) https://orcid.org/0000-0001-5118-1313

A. Karim (iD https://orcid.org/0000-0002-8414-9579

K. K. Knudsen (iD https://orcid.org/0000-0002-7821-8873

J. L. Wardlow (iD https://orcid.org/0000-0003-2376-8971

A. Weiß (iD https://orcid.org/0000-0003-4678-3939

P. P. van der Werf (iD https://orcid.org/0000-0001-5434-5942

\section{References}

Alaghband-Zadeh, S., Chapman, S. C., Swinbank, A. M., et al. 2012, MNRAS, 424, 2232

ALMA Partnership, Vlahakis, C., Hunter, T. R., et al. 2015, ApJL, 808, L4

Astropy Collaboration, Robitaille, T. P., Tollerud, E. J., et al. 2013, A\&A, 558, A33

Bacon, R., Copin, Y., Monnet, G., et al. 2001, MNRAS, 326, 23

Barger, A. J., Cowie, L. L., Chen, C.-C., et al. 2014, ApJ, 784, 9

Barger, A. J., Cowie, L. L., Sanders, D. B., et al. 1998, Natur, 394, 248

Barger, A. J., Wang, W.-H., Cowie, L. L., et al. 2012, ApJ, 761, 89

Baugh, C. M., Lacey, C. G., Frenk, C. S., et al. 2005, MNRAS, 356, 1191

Begeman, K. G. 1989, A\&A, 223, 47

Bolatto, A. D., Wolfire, M., \& Leroy, A. K. 2013, ARA\&A, 51, 207

Bothwell, M. S., Chapman, S. C., Tacconi, L., et al. 2010, MNRAS, 405, 219

Bothwell, M. S., Smail, I., Chapman, S. C., et al. 2013, MNRAS, 429, 3047

Bouché, N., Cresci, G., Davies, R., et al. 2007, ApJ, 671, 303

Bournaud, F., Perret, V., Renaud, F., et al. 2014, ApJ, 780, 57

Bouwens, R. J., Illingworth, G. D., Oesch, P. A., et al. 2015, ApJ, 803, 34

Brammer, G. B., van Dokkum, P. G., Franx, M., et al. 2012, ApJS, 200, 13

Buat, V., Iglesias-Páramo, J., Seibert, M., et al. 2005, ApJL, 619, L51

Burkert, A., Genzel, R., Bouché, N., et al. 2010, ApJ, 725, 2324

Calanog, J. A., Fu, H., Cooray, A., et al. 2014, ApJ, 797, 138

Casey, C. M., Narayanan, D., \& Cooray, A. 2014a, PhR, 541, 45

Casey, C. M., Scoville, N. Z., Sanders, D. B., et al. 2014b, ApJ, 796, 95

Chabrier, G. 2003, PASP, 115, 763

Chapman, S. C., Blain, A. W., Smail, I., \& Ivison, R. J. 2005, ApJ, 622, 772

Chen, C.-C., Smail, I., Ivison, R. J., et al. 2016, ApJ, 820, 82

Chen, C.-C., Smail, I., Swinbank, A. M., et al. 2015, ApJ, 799, 194

Courteau, S. 1997, AJ, 114, 2402

Cowley, W. I., Lacey, C. G., Baugh, C. M., \& Cole, S. 2015, MNRAS, 446, 1784

da Cunha, E., Walter, F., Smail, I. R., et al. 2015, ApJ, 806, 110

Daddi, E., Elbaz, D., Walter, F., et al. 2010, ApJL, 714, L118

Danielson, A. L. R., Swinbank, A. M., Smail, I., et al. 2011, MNRAS, 410, 1687

Danielson, A. L. R., Swinbank, A. M., Smail, I., et al. 2013, MNRAS, 436, 2793

Danielson, A. L. R., Swinbank, A. M., Smail, I., et al. 2017, ApJ, 840, 78

Davé, R., Finlator, K., Oppenheimer, B. D., et al. 2010, MNRAS, 404, 1355

De Breuck, C., Williams, R. J., Swinbank, M., et al. 2014, A\&A, 565, A59

Decarli, R., Walter, F., Aravena, M., et al. 2016, ApJ, 833, 70

Downes, D., \& Solomon, P. M. 1998, ApJ, 507, 615

Engel, H., Tacconi, L. J., Davies, R. I., et al. 2010, ApJ, 724, 233

Foreman-Mackey, D., Hogg, D. W., Lang, D., \& Goodman, J. 2013, PASP, 125,306

Förster Schreiber, N. M., Genzel, R., Bouché, N., et al. 2009, ApJ, 706, 1364
Fu, H., Cooray, A., Feruglio, C., et al. 2013, Natur, 498, 338

Genzel, R., Schreiber, N. M. F., Übler, H., et al. 2017, Natur, 543, 397

Genzel, R., Tacconi, L. J., Gracia-Carpio, J., et al. 2010, MNRAS, 407, 2091 Gil de Paz, A., Boissier, S., Madore, B. F., et al. 2007, ApJS, 173, 185

Ginolfi, M., Maiolino, R., Nagao, T., et al. 2017, MNRAS, 468, 3468

Greisen, E. W., Calabretta, M. R., Valdes, F. G., \& Allen, S. L. 2006, A\&A, 446, 747

Grogin, N. A., Kocevski, D. D., Faber, S. M., et al. 2011, ApJS, 197, 35

Gunn, J. E., Siegmund, W. A., Mannery, E. J., et al. 2006, AJ, 131, 2332

Harris, A. I., Baker, A. J., Zonak, S. G., et al. 2010, ApJ, 723, 1139

Harrison, C. M., Alexander, D. M., Swinbank, A. M., et al. 2012, MNRAS, 426, 1073

Hayward, C. C., Narayanan, D., Kereš, D., et al. 2013, MNRAS, 428, 2529

Hodge, J. A., Carilli, C. L., Walter, F., et al. 2012, ApJ, 760, 11

Hodge, J. A., Karim, A., Smail, I., et al. 2013, ApJ, 768, 91

Hodge, J. A., Riechers, D., Decarli, R., et al. 2015, ApJL, 798, L18

Hodge, J. A., Swinbank, A. M., Simpson, J. M., et al. 2016, ApJ, 833, 103

Hopkins, P. F., Cox, T. J., Hernquist, L., et al. 2013, MNRAS, 430, 1901

Howell, J. H., Armus, L., Mazzarella, J. M., et al. 2010, ApJ, 715, 572

Hughes, D. H., Serjeant, S., Dunlop, J., et al. 1998, Natur, 394, 241

Hung, C.-L., Rich, J. A., Yuan, T., et al. 2015, ApJ, 803, 62

Hurvich, C. M., \& Tsai, C.-L. 1989, Biometrika, 76, 297

Huynh, M. T., Emonts, B. H. C., Kimball, A. E., et al. 2017, MNRAS, 467, 1222

Ikarashi, S., Ivison, R. J., Caputi, K. I., et al. 2015, ApJ, 810, 133

Iono, D., Wilson, C. D., Takakuwa, S., et al. 2007, ApJ, 659, 283

Ivison, R. J., Papadopoulos, P. P., Smail, I., et al. 2011, MNRAS, 412, 1913

Karim, A., Swinbank, A. M., Hodge, J. A., et al. 2013, MNRAS, 432, 2

Kennicutt, R. C., Calzetti, D., Aniano, G., et al. 2011, PASP, 123, 1347

Kennicutt, R. C., \& Evans, N. J. 2012, ARA\&A, 50, 531

Kennicutt, R. C., Jr. 1989, ApJ, 344, 685

Kennicutt, R. C., Jr. 1998a, ARA\&A, 36, 189

Kennicutt, R. C., Jr. 1998b, ApJ, 498, 541

Kennicutt, R. C., Jr., Armus, L., Bendo, G., et al. 2003, PASP, 115, 928

Koekemoer, A. M., Faber, S. M., Ferguson, H. C., et al. 2011, ApJS, 197, 36

Kong, X., Charlot, S., Brinchmann, J., \& Fall, S. M. 2004, MNRAS, 349, 769

Koprowski, M. P., Coppin, K. E. K., Geach, J. E., et al. 2016, ApJL, 828, L21

Krajnović, D., Cappellari, M., de Zeeuw, P. T., \& Copin, Y. 2006, MNRAS, 366, 787

Leroy, A. K., Bolatto, A., Gordon, K., et al. 2011, ApJ, 737, 12

Leroy, A. K., Walter, F., Bigiel, F., et al. 2009, AJ, 137, 4670

Leroy, A. K., Walter, F., Brinks, E., et al. 2008, AJ, 136, 2782

Madau, P., \& Dickinson, M. 2014, ARA\&A, 52, 415

Menéndez-Delmestre, K., Blain, A. W., Swinbank, M., et al. 2013, ApJ, 767, 151

Meurer, G. R., Heckman, T. M., \& Calzetti, D. 1999, ApJ, 521, 64

Miller, S. H., Bundy, K., Sullivan, M., Ellis, R. S., \& Treu, T. 2011, ApJ, 741,115

Molina, J., Ibar, E., Swinbank, A. M., et al. 2017, MNRAS, 466, 892

Narayanan, D., Dave, R., Johnson, B., et al. 2017, MNRAS, submitted (arXiv:1705.05858)

Narayanan, D., Hayward, C. C., Cox, T. J., et al. 2010, MNRAS, 401, 1613

Olivares, V., Treister, E., Privon, G. C., et al. 2016, ApJ, 827, 57

Osterbrock, D. E. 1989, Astrophysics of Gaseous Nebulae and Active Galactic Nuclei (Mill Valley, CA: Univ. Science Books)

Overzier, R. A., Heckman, T. M., Wang, J., et al. 2011, ApJL, 726, L7

Pettini, M., \& Pagel, B. E. J. 2004, MNRAS, 348, 59

Planck Collaboration, Ade, P. A. R., Aghanim, N., et al. 2014, A\&A, 571, A16

Popping, G., Puglisi, A., \& Norman, C. A. 2017, MNRAS, submitted (arXiv:1706.06587)

Rawle, T. D., Egami, E., Bussmann, R. S., et al. 2014, ApJ, 783, 59

Reddy, N., Dickinson, M., Elbaz, D., et al. 2012, ApJ, 744, 154

Riechers, D. A., Hodge, J., Walter, F., Carilli, C. L., \& Bertoldi, F. 2011, ApJL, 739, L31

Rousselot, P., Lidman, C., Cuby, J.-G., Moreels, G., \& Monnet, G. 2000 , A\&A, 354, 1134

Sakamoto, K., Ho, P. T. P., \& Peck, A. B. 2006, ApJ, 644, 862

Sanders, D. B., \& Mirabel, I. F. 1996, ARA\&A, 34, 749

Sandstrom, K. M., Leroy, A. K., Walter, F., et al. 2013, ApJ, 777, 5

Schmidt, M. 1959, ApJ, 129, 243

Scoville, N. Z., Sargent, A. I., Sanders, D. B., \& Soifer, B. T. 1991, ApJL, 366, L5

Scoville, N. Z., Yun, M. S., \& Bryant, P. M. 1997, ApJ, 484, 702

Shapiro, K. L., Genzel, R., Förster Schreiber, N. M., et al. 2008, ApJ, 682, 231

Sharon, C. E., Baker, A. J., Harris, A. I., \& Thomson, A. P. 2013, ApJ, 765, 6

Sharon, C. E., Riechers, D. A., Hodge, J., et al. 2016, ApJ, 827, 18 
Simpson, J. M., Smail, I., Swinbank, A. M., et al. 2015, ApJ, 799, 81 Simpson, J. M., Smail, I., Swinbank, A. M., et al. 2017, ApJ, 839, 58 Simpson, J. M., Swinbank, A. M., Smail, I., et al. 2014, ApJ, 788, 125 Siringo, G., Kreysa, E., Kovács, A., et al. 2009, A\&A, 497, 945

Skelton, R. E., Whitaker, K. E., Momcheva, I. G., et al. 2014, ApJS, 214, 24 Smail, I., Ivison, R. J., \& Blain, A. W. 1997, ApJL, 490, L5

Solomon, P. M., \& Vanden Bout, P. A. 2005, ARA\&A, 43, 677

Spilker, J. S., Aravena, M., Marrone, D. P., et al. 2015, ApJ, 811, 124

Spilker, J. S., Marrone, D. P., Aguirre, J. E., et al. 2014, ApJ, 785, 149

Spilker, J. S., Marrone, D. P., Aravena, M., et al. 2016, ApJ, 826, 112

Swinbank, A. M., Chapman, S. C., Smail, I., et al. 2006, MNRAS, 371, 465

Swinbank, A. M., Simpson, J. M., Smail, I., et al. 2014, MNRAS, 438, 1267

Swinbank, A. M., Smail, I., Chapman, S. C., et al. 2010, MNRAS, 405, 234

Swinbank, A. M., Sobral, D., Smail, I., et al. 2012, MNRAS, 426, 935
Tacconi, L. J., Genzel, R., Smail, I., et al. 2008, ApJ, 680, 246

Tacconi, L. J., Neri, R., Genzel, R., et al. 2013, ApJ, 768, 74

Tadaki, K.-i., Kodama, T., Nelson, E. J., et al. 2017, ApJL, 841, L25

Takeuchi, T. T., Yuan, F.-T., Ikeyama, A., Murata, K. L., \& Inoue, A. K. 2012, ApJ, 755, 144

Thomson, A. P., Ivison, R. J., Owen, F. N., et al. 2015, MNRAS, 448, 1874

To, C.-H., Wang, W.-H., \& Owen, F. N. 2014, ApJ, 792, 139

Ueda, J., Iono, D., Yun, M. S., et al. 2014, ApJS, 214, 1

van der Wel, A., Franx, M., van Dokkum, P. G., et al. 2014, ApJ, 788, 28

Walter, F., Brinks, E., de Blok, W. J. G., et al. 2008, AJ, 136, 2563

Wang, S. X., Brandt, W. N., Luo, B., et al. 2013, ApJ, 778, 179

Wardlow, J. L., Smail, I., Coppin, K. E. K., et al. 2011, MNRAS, 415, 1479

Weiß, A., Kovács, A., Coppin, K., et al. 2009, ApJ, 707, 1201

Younger, J. D., Fazio, G. G., Wilner, D. J., et al. 2008, ApJ, 688, 59 